\title{
FEASIBILITY OF INTERSTITIAL \\ EX-VIVO MAMMARY \\ AUTOFLUORESCNE \\ MICROENDOSCOPY
}

\author{
By: \\ Dena Monjazebi \\ B.Sc. BIHE, 2011 \\ Tehran, Iran
}

A thesis

presented to Ryerson University in partial

fulfillment of the requirements for

the degree of Master of Science

in the Program of Biomedical Physics

Toronto, Ontario, Canada, 2016

(C) Dena Monjazebi 2016 


\section{Author's Declaration}

I hereby declare that I am the sole author of this thesis. This is a true copy of the thesis, including any required final revisions as accepted by my examiners.

I authorize Ryerson University to lend this thesis to other institutions or individuals for the purpose of scholarly research.

I further authorize Ryerson University to reproduce this thesis by photocopying or by other means, in total or in part, at the request of other institutions or individuals for the purpose of scholarly research.

I understand that this thesis may be made electronically available to the public.

Dena Monjazebi 


\title{
Abstract
}

\section{Feasibility of Interstitial Ex-vivo Mammary Autofluorescence Microendoscopy}

\author{
Dena Monjazebi \\ Master of Science \\ Biomedical Physics, 2016 \\ Ryerson University
}

In the past decades our knowledge of breast cancer has been rapidly evolving yet the basic paradigm of diagnosis and treatment of cancer has not. In cancer diagnosis, presentation of breast cancer can be a palpable lump or a suspicious mass on screening imaging, namely a mammogram. However, malignancy will be ascertained by tissue biopsy if needed. Biopsy is the gold standard breast cancer diagnostic test. Biopsy sampling is invasive, painful and costly. In addition, when the interpretation of current imaging modalities is not concordant with pathology results the biopsies may have to be repeated. Microendoscopy autofluorescence (AM) is a method of acquiring images directly from the tissues that contain fluorescent susceptible molecules (fluorophore). Studies of endoscopy in colon and esophagus showed that AM imaging is capable to recognize malignancy and can be utilized to discriminate between normal tissue and tumor. Additionally, it has been shown that, AM was able to differentiate cancer versus normal cells when a microendoscope was inserted into a breast duct. The main purpose of this study is to investigate if the same contrast exists if AM applied interstitially into the ex-vivo mastectomy breast tissues. This is a feasibility study to explore if interstitial AM has the potential to be coupled with breast cancer imaging diagnostics to provide better discrimination of the characteristics of the target tissue inside. The success in this approach could significantly reduce 
the number of required tissue biopsies to confirm the diagnosis. 


\section{Acknowledgements}

I would like to express my special thanks of gratitude to my supervisors Dr. Alexandre Douplik and Dr. Alexandra Easson for their guidance, understanding and encouragement, who gave me the golden opportunity to do this wonderful project on the topic. For everything you've done for me, I thank you.

I am also thankful to my committee members Dr. Carl Kumaradas and Dr. Valdislav Toronov. I thank their paramount mentorship in providing a well-rounded experience during my graduate studies.

I would like to thank the Department of physics at Ryerson University, especially Dr. Pedro Goldman for his beneficial comments on my thesis. I also wish to thank Adriana Gaertner and Maryrose Tait for their overwhelming support and guidance. I thank Arthur Worthington and Graham Pearson without whom this research would have been so lacking

Finally, and most importantly, I would like to thank my family members, Aida, Behnam Simin and Tala. Their support, encouragement, quiet patience and unwavering love were undeniably the bedrock upon which the past years of my life have been built. It was under their watchful eye that I gained so much drive and an ability to tackle challenges head on. 


\section{Table of Contents}

Author's Declaration......................................... ii

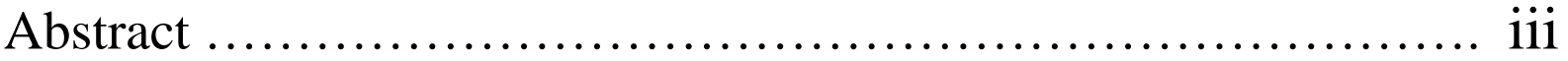

Acknowledgements ................................... iv

Table of Contents ..................................... v

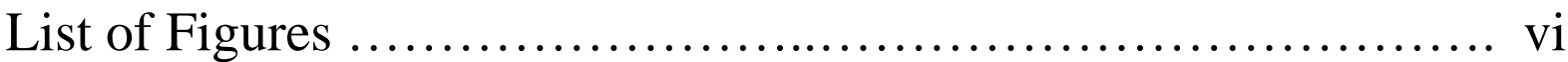

List of Abbreviations...........................................................

Chapter 1: Introduction ........................................................... 1

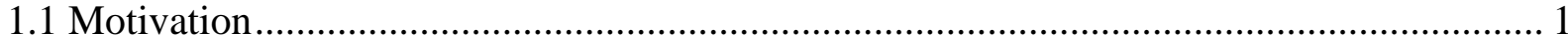

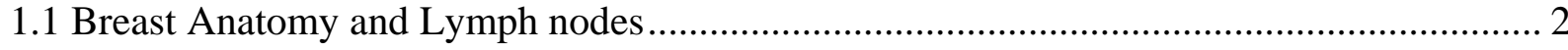

1.2 Breast Cancer and Diagnostic methods ........................................................................... 3

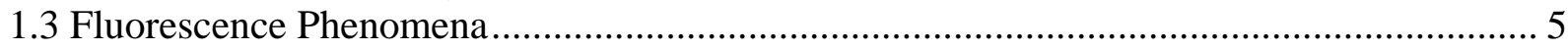

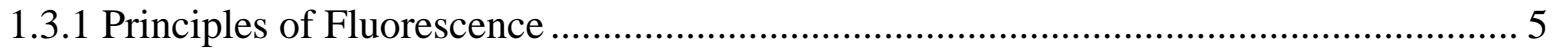

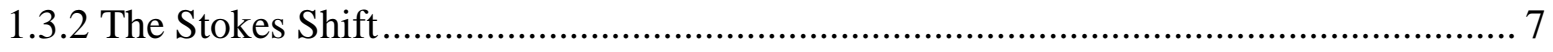

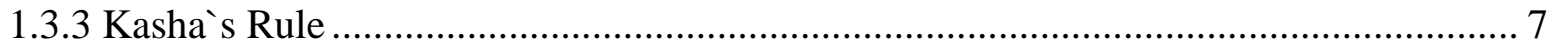

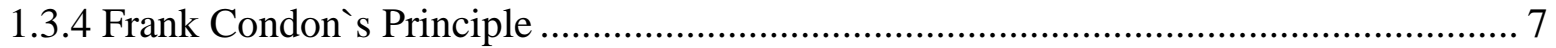

1.4 Autofluorescence Microendoscopy Imaging ................................................................. 9

1.4.1 Endogenous Sources of Autofluorescence and Origins of Normal-malignant Contrast 9

1.4.2 Autofluorescence Endoscopy ..................................................................................... 11

1.4.3 Shortcomings in Breast Cancer Diagnostic Methods ............................................... 12

1.4.4 Ductoscopy vs. Interstitial Microendoscopy.............................................................. 14

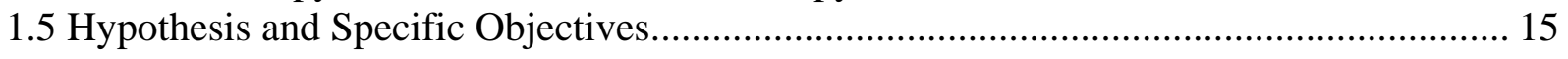

Chapter 2: Materials and Methods ................................................ 17

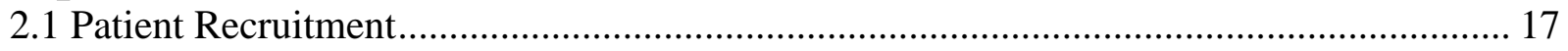

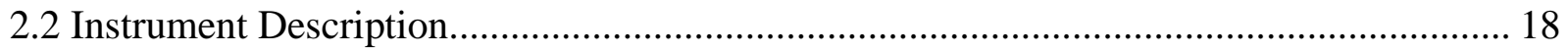

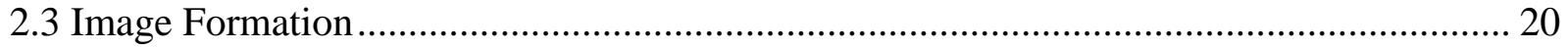

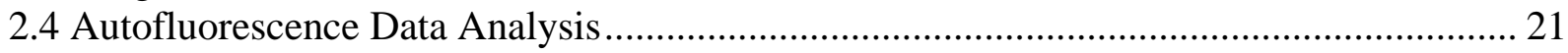

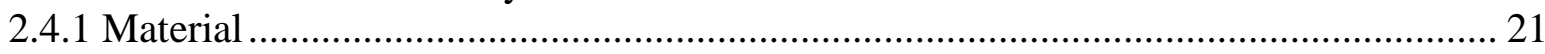

2.4.2 Image Processing .................................................................................................. 21

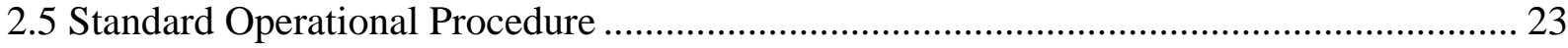

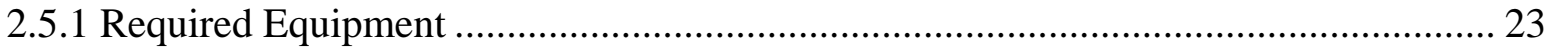

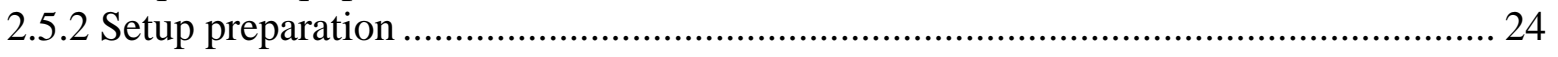

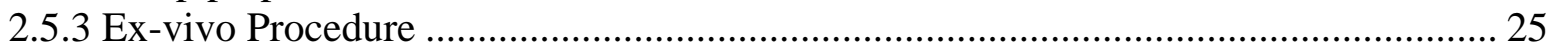

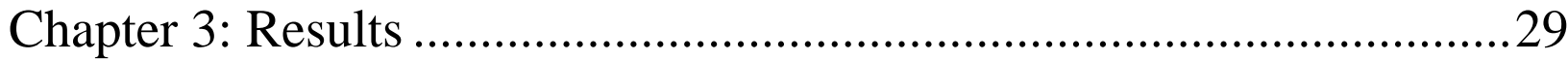

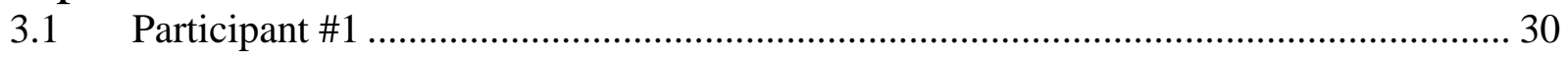

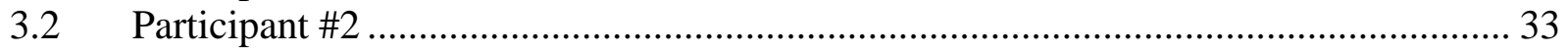

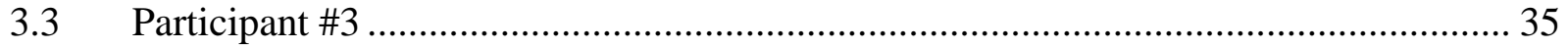

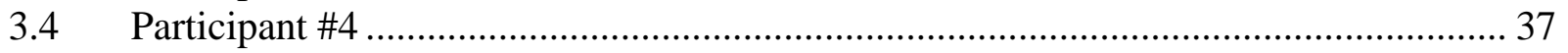




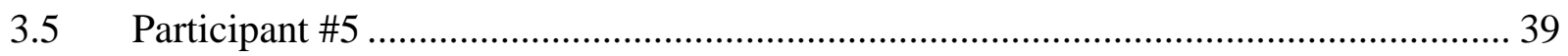

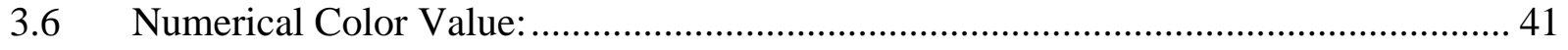

Chapter 4: Discussion and Conclusion.............................................44

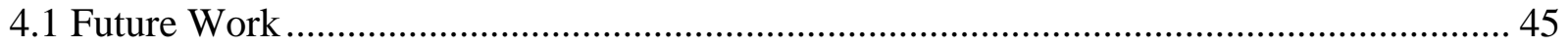

Bibliography ..........................................................................47 


\section{List of Tables}

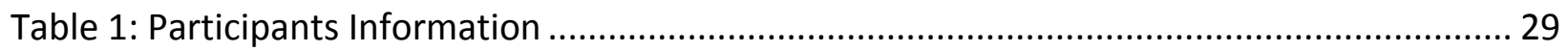

Table 2: Pathology Assessment of Samples acquired for correlation purposes in the study for participant No.1 ....................................................................................................... 32

Table 3: Pathology Assessment of Samples acquired for correlation purposes in the study for participant No.2

Table 4: Pathology Assessment of Samples acquired for correlation purposes in the study for participant No.3 ....................................................................................................... 36

Table 5: Pathology Assessment of Samples acquired for correlation purposes in the study for participant No.4 ……............................................................................................ 38

Table 6: Pathology Assessment of Samples acquired for correlation purposes in the study for

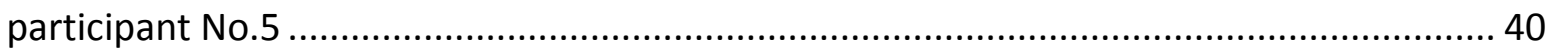

Table 7: Average, maximum and minimum of all 5 cases NCVs for normal versus cancer.......... 42 


\section{List of Figures}

Figure 1: Schematic showing human breast components [13] ................................................... 3

Figure 2: Jablonski diagram, an illustration of fluorescence and phosphorescence [23] ............. 5

Figure 3: An illustration of an absorption and emission spectrum and Stokes shift................... 7

Figure 4: Franck-Condon principle energy diagram .......................................................... 8

Figure 5: Representation of the absorption and fluorescence spectra corresponding to the

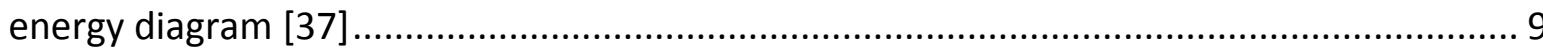

Figure 6: Absorption and emission spectra of important endogenous fluorophores [40]. ......... 10 Figure 7: Sources of contrast a) An illustration of collagen modification upon cancer growth that reduce the intesity of autofluorescence $b$ ) Tumor has highier absorption with respect to healthy and therefore absorbs both excitation and fluorescence lights more.................. 11

Figure 8: White light(left) and autofluorescence (right) endoscopic images of dysplasia colon.

The autofluorescence images shows a clear cancer-malignant contrast [56]................... 12

Figure 9: a) White-light imaging of Type Ilc esophageal cancer in the posterior wall of the lower esophagus. (b) On autofluorescence view, the lesion could be located as darker areas [59].

12

Figure 10: Ultrasound-guided breast biopsy [64] ............................................................ 13

Figure 11: Comparative presentation of normal region[ $a$ white light and $b$ autofluorescence] and tumor region [c white light and d autofluorescence] [6] ...................................... 15

Figure 12: Schematic representation of autofluorescence ductoscopy (left) and Interstitial

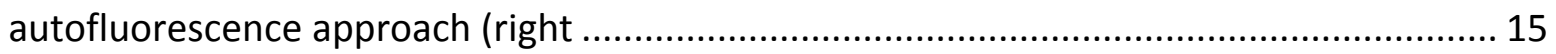

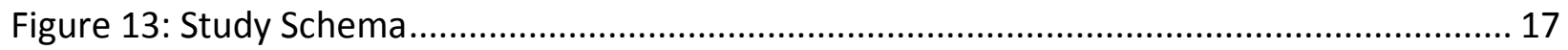

Figure 14: Schematic representation of setup ................................................................... 18

Figure 15: Microendoscope ................................................................................................ 19

Figure 16: White light (left) and autofluorescence (right) images of USADF 1951 target .......... 19

Figure 17: Blue and red illumination pathways and autofluorescence image formation ........... 20

Figure 18: Autofluorescence image acquired from normal fresh pork fat specimen................. 21

Figure 19: Autofluorescence image of fresh lamb liver (a) - Line plot of $R, G$ and $B$ channels along

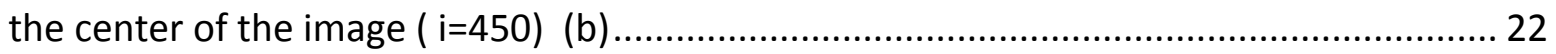

Figure 20: Autofluorescence image of fresh lamb liver containing relatively larger amount of blood (a) - Line plot of $R, G$ and $B$ channels along the center of the image(b) ................... 23

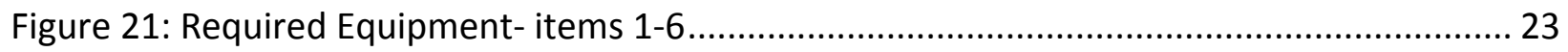

Figure 22: Required Equipment - LuerLock ............................................................................ 23

Figure 23: Required equipment-modified trocar .............................................................. 24

3) Figure 24: Required Equipment- Magnum gun for acquiring biopsies............................ 24

Figure 25: Required Equipment - Short Handle..................................................................... 24

Figure 26: Required Equipment- Data Acquisition setup ................................................... 25

Figure 27: Procedure- Mastectomy specimen ................................................................... 25

Figure 28: Procedure- Trocar Insertion. Cross signifies the location of tumor. ........................ 26

Figure 29: Procedure- clear interstitial channel ............................................................. 26

Figure 30: Procedure- Saline injection and Data Acquisition .............................................. 27

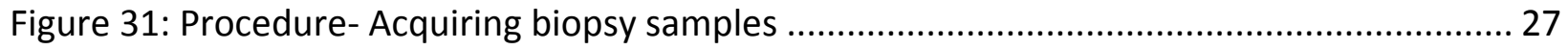


Figure 32: Procedure- Core-needle Biopsy Sample.....

Figure 33: Data acquisition- Participant no. 1. Cross signifies the location of the tumor............ 30 Figure 34: Case 1: Normal (a) white light and (b) autofluorescence compared to tumor (c) white light and (d) autofluorescence. The field of view has a diameter of approximately $2 \mathrm{~mm}$. The NCV locations and related values are shown in $b$ and $d$.

Figure 35: Case 1: Line plot of green, red and blue channels of autofluorescence images along

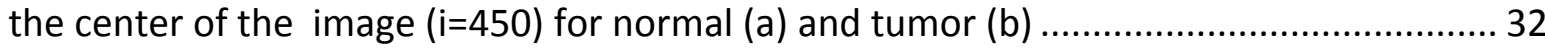

Figure 36: Data acquisition- Participant no. 2- cross signifies the location of the tumor ............ 33 Figure 37: Case 2: Autofluorescence imaging of normal (a) versus malignant tumor (b) . The field of view has a diameter of approximately $2 \mathrm{~mm}$. The NCV locations and related values are shown.

Figure 38: Case 2: Line plot of green, red and blue channels of autofluorescence images along the center of the image $(\mathrm{i}=450)$ for normal $(\mathrm{a})$ and tumor $(\mathrm{b})$........................................ 34

Figure 39: Data acquisition- Participant no. 3. Cross signifies the location of the tumor........... 35 Figure 40: Case 3: Autofluorescence imaging of normal (a) versus malignant tumor (b) . The field of view has a diameter of approximately $2 \mathrm{~mm}$. The NCV locations and related values are shown.

Figure 41: Case 3: Line plot of green, red and blue channels of autofluorescence images along

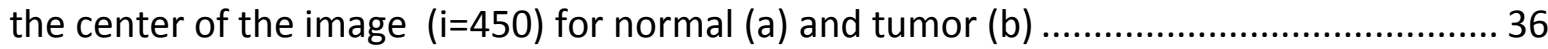

Figure 42: Data acquisition- Participant no. 4. Cross signifies the location of the tumor........... 37 Figure 43: Case 4: Autofluorescence imaging of normal (a) versus malignant tumor (b) . The field of view has a diameter of approximately $2 \mathrm{~mm}$. The NCV locations and related values are shown.

Figure 44: Case 4: Line plot of green, red and blue channels of autofluorescence images along

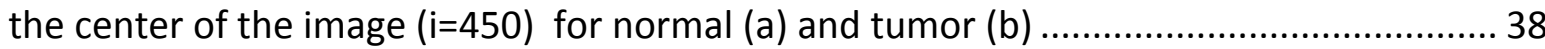

Figure 45: Data acquisition- Participant no. 5. Cross signifies the location of the tumor............ 39 Figure 46: Case 5: Autofluorescence imaging of normal (a) versus malignant tumor (b) . The field of view has a diameter of approximately $2 \mathrm{~mm}$. The NCV locations and related values are shown.

Figure 47: Case 5: Line plot of green, red and blue channels of autofluorescence images along the center of the image $(i=450)$ for normal (a) and tumor (b) .

Figure 48: A comparison between the average NCVs of normal and malignant for all 5 cases and error bars 


\section{List of Abbreviations}

AF: Autofluorescence

AM: Autofluorescence Microendoscope

WL: White light

ER: Estrogen receptor

PR: Progesterone receptor

HER2: Human epidermal growth factor receptor

IDC: Invasive Ductal Carcinoma 


\section{Chapter 1: Introduction}

\subsection{Motivation}

Breast Cancer is the most common cancer among Canadian women with the exception of non-melanoma skin cancer and is responsible for $15 \%$ of all cancer related deaths in women [1]. Once a breast abnormality is found by screening or clinically, depending on the degree of suspicion of cancer, a core biopsy is done to confirm whether the tissue is benign or malignant. The present imaging modalities namely, mammography and ultrasonography play a vital role in aiding clinicians to an accurate diagnosis and to characterize lesions with the purpose of selecting the most efficient treatment plan. Despite the advances in diagnostic imaging in recent years which led to an increase in cancer survival by $20-30 \%$ [2], there exists an area of radiological indeterminacy where the standard diagnostic methods cannot discriminate between benign and malignant lesions. Among all screening mammograms, 5\% to $9 \%$ are considered ambiguous [3]. The recommended approach is a frequent follow up or biopsy. Biopsy is the gold standard diagnostic tool. However, when the core biopsy is not concordant with diagnostic image interpretation, the biopsy sampling may need to be repeated. Although mammography and ultrasonography are the most robust no-cut diagnostic methods, in some cases they lack the accuracy to clarify the diagnostic ambiguities. Currently there is no practical way of looking directly at the tissue with the ability to clarify the status of questionable abnormalities and to avoid unnecessary biopsies.

Autofluorescence (AF) imaging was previously used to differentiate between normal and abnormal tissue seen via endoscopy in the colon and lung [4]. A combination of AF and multi-spectral imaging was needed in the esophagus to guide biopsies [5]. Standard white light was also previously employed for microendoscopic exploration of breast cancer through ducts along with AF imaging of the tissue of interest recorded at 30 frames/sec. The developed device was designed such that abundant fluorophores of breast healthy tissue would be illuminated. In that study, cancer was found to have less green AF relative to normal tissue when excited by blue light, so in the false color image the normal tissue appears cyan, while abnormal tissue has a range of red color in each pixel, 
depending on the red-to-green ratio [5] [6] [7] [8]. A comparison between AF imaging and standard white light microendoscopy depicts that the former delineates malignancy more precisely. While malignancy may be noticeable as protrusions under white light imaging a distinctive color boundary between cancer and normal was observed with $\mathrm{AF}$ microendocopy [9].

Mammary Microendoscopy (MM) allows a closer look of the breast tumor. Direct insertion of the MM into the nipple orifice, (i.e. ductoscopy), not only allows the mammary ductal epithelium to be directly visualized, but also biopsy and cytological analysis of intraductal lesions can be performed via this method. [10] [11]. Previous studies reported that although ductoscopy is easy and practical, its application in breast cancer management is limited [12]. In this pilot prospective clinical study, an alternative approach with potentially more practicable applications was tested. The goal was to investigate if the Autofluorescence Microendoscope (AM) was capable of recognizing malignancy when the suspicious tissue was reached interstitially. This study integrated the advantages of autofluorescence imaging with interstitial MM to examine its potential to guide breast cancer biopsies. AM has never been applied outside the duct. For the future, AM can also be applied in-vivo. The ultimate aim in this study was to provide the diagnostic radiologist with an additional tool to help differentiate between benign and malignant tissue, and thus improve the accuracy and reduce the numbers of required biopsies. In this study the same device that has been developed and employed in the ductoscopy study, was coupled to a proper AM.

\subsection{Breast Anatomy and Lymph nodes}

The female breast is a mass of epithelial, adipose (fat) and fibrous tissues and is located over pectoral muscles of the chest wall. The breast is an organ specific for milk production and consists of components that support its function. The fatty tissue forms the main bulk of the breast and house the milking glands. The epithelial part of the breast is composed of lobules, glands that produce milk and ducts that transfer milk to the nipple reservoir [13]. Most cancers of the breast arise from the cells that form the lobules and terminal ducts [14]. The male breast is almost analogous to that of the female breast, however, as there is no physiologic need for lactation, the male breast has no lobules. 


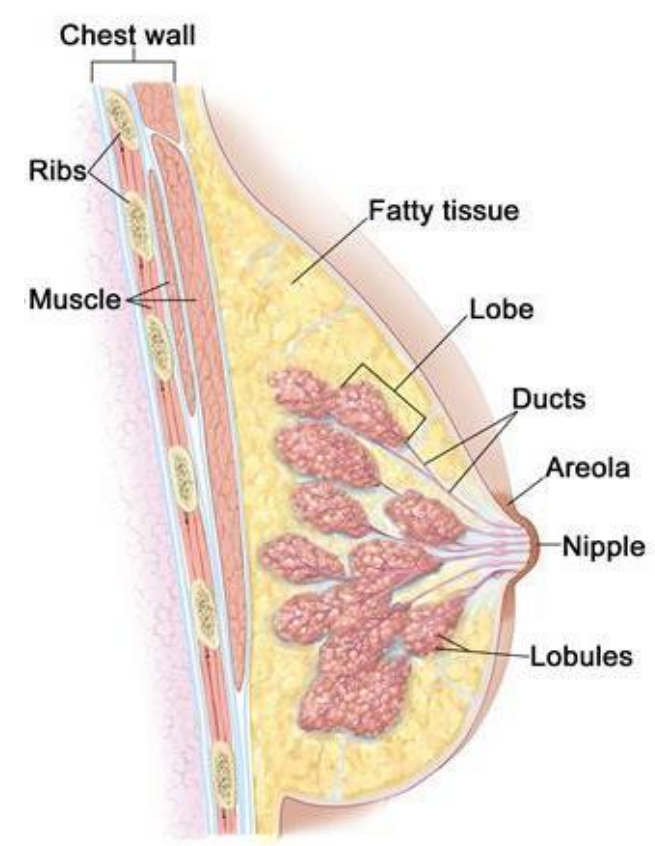

Figure 1: Schematic showing human breast components [13]

Lymph nodes are kidney or oval shaped and vary in size from a few millimeters to about 1-2 cm long [15]. Lymph nodes are connected by lymphatic vessels and distributed broadly throughout the body while armpit and groin are areas with relatively higher concentration. Lymph nodes are main places of B, T and other immune cells and also acts as filters for foreign elements and cancer cells. They become inflamed or enlarged in various diseases like infections or cancer invasion.

\subsection{Breast Cancer and Diagnostic methods}

Breast cancer is the most common form of malignancy among women, a potentially curable disease by means of various treatment methods. Breast cancer generally occurs in women, but can develop in men. Death from breast cancer is the second leading cause of cancer death in women, only lung cancer causes more deaths [2]. The lifetime prevalence of invasive breast cancer in North America women is $12 \%$ ( 1 in 8 women) and is the second most prevalence cancer in the US [16]. The median age at death from breast cancer was 68 years of age [17]. However, breast cancer mortality rates have been in decline due to better treatment options and earlier detection methods [18]. Many risk 
factors increase the chance of developing breast cancer including genetic, hormonal and environmental influences [16]. The strongest risk factor for breast cancer is age, with increased risk associated with invasive breast cancer in women 55 and older [2].

The development of invasive breast cancer in humans is thought to occur over a number of years through a progression of abnormal processes. The delineated sequential stages of this course are commonly referred to as hyperplasia, atypical hyperplasia, carcinoma in situ, and invasive breast cancer. Carcinoma in situ may be lobular (lobular carcinoma in situ) or ductal (ductal carcinoma in situ [DCIS]) in origin and can be graded histologically from low-to high-grade according to decreasing differentiation of the tumor. DCIS, the most common type of in situ breast carcinoma, now comprises $20 \%$ to $25 \%$ of all diagnosed breast tumors compared with $<5 \%$ of diagnosed breast cancer cases before the 1980s, this is largely because of the increase in mammography screening [18] [19].

Patients with positively malignant findings or suspicious results, such as a palpable lump or an abnormal result from a screening mammography, will undergo further diagnostic evaluations. This involves multiple components, including assessment of the patient's medical history and family history of cancer, physical examination, additional mammographic and diagnostic ultrasound imaging, and, for some patients, screening magnetic resonance imaging (MRI) [20]. Eventually based on the findings from diagnostic imaging, biopsy sampling may be required to confirm the radiologic diagnosis of benign or malignant tissue. Diagnosis and staging of breast cancer ultimately depends on the pathological evaluation of biopsy samples. Staging of the cancer allows clinicians to select the most appropriate treatment among the wide variety of therapies available for breast cancer. The major prognostic indicators of cancer recurrence or death from breast cancer are age, tumor size, tumor grade, comorbidities, and the number of axillary lymph nodes affected [21]. Knowledge of an individual's risk factor for breast cancer can be useful for deciding whether the harms associated with mammography, such as falsepositive tests and unnecessary early treatment is outweighed by the patient`s risk status.

Today, breast cancer treatment is multi-dimensional and therapeutic options include surgery, radiation therapy, chemotherapy, hormonal therapy and the latest targeted therapies ,e.g., monoclonal antibodies drugs to treat HER2-positive breast cancer. 
Surgical options include segmental mastectomy and lumpectomy followed by a course of radiation therapy or total mastectomy depending on multiple clinical factors [22].

\subsection{Fluorescence Phenomena}

\subsubsection{Principles of Fluorescence}

Electronic excitation of susceptible molecules by light (absorption) is followed by returning of the molecule to its ground state and emission of light with a longer wavelength with respect to the absorbed light after a brief interval, this interval is known as the fluorescence lifetime (around $10^{9}$ seconds). The probability of this process is described by its quantum yield and depends on the chemical structure and its environment. This phenomenon is called photoluminescence which depending on the emission pathway is formally divided into subcategories, fluorescence and phosphorescence.

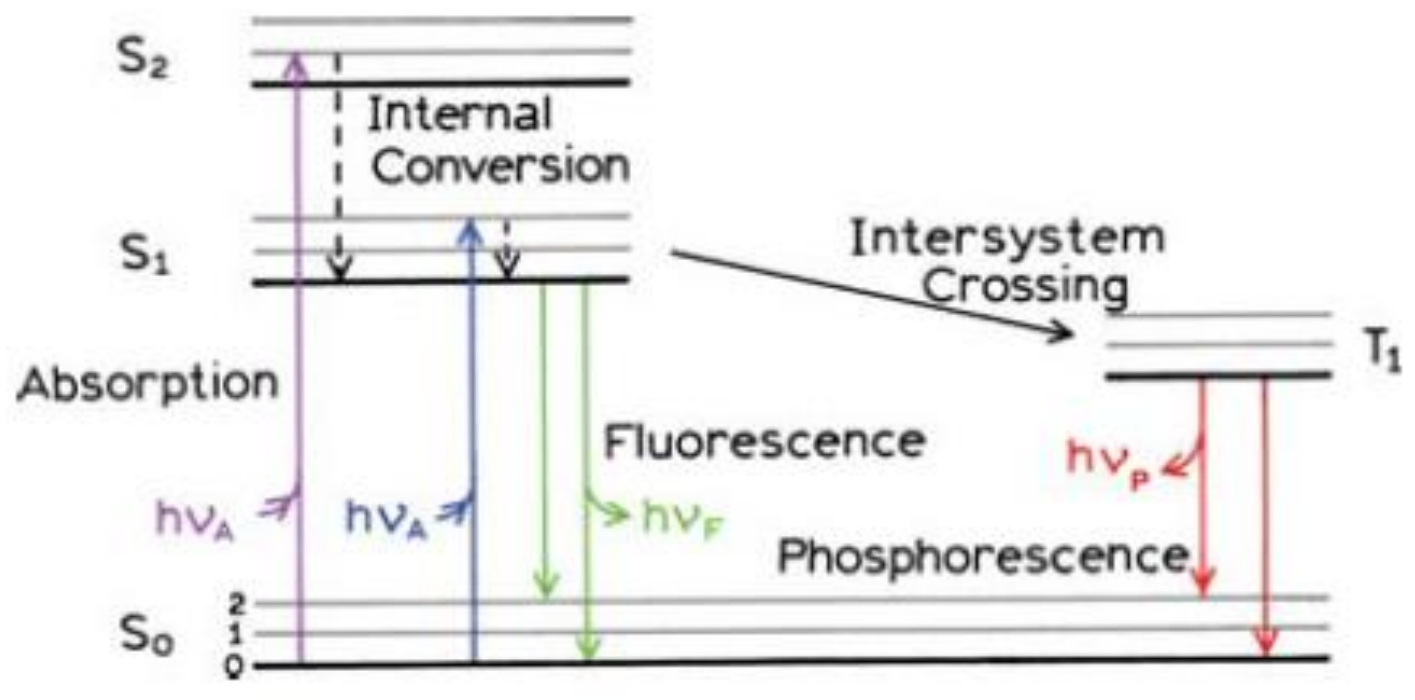

Figure 2: Jablonski diagram, an illustration of fluorescence and phosphorescence [23]

The fluorophore is excited to a higher energy level of $S_{1}$ or $S_{2}$ (The first and second excited states). Immediately succeeding the absorption of a photon, several processes will occur with various probabilities (figure 2). However, the dominant mechanism is a nonradiative relaxation to the lowest vibrational energy level of $S_{1}$ which has the same spin multiplicity. The excess of the vibrational energy is converted into IR radiation. This 
process is termed as internal conversion or vibrational relaxation and generally transpires in picoseconds or less [24] [23].

Electrons can undergo two pathways to return to the ground state. A molecular electronic state of a system that all electron spins are paired is called singlet state [25]. Molecules in singlet energy state $S_{1}$ can return to the ground state upon emitting photon. This spin-allowed emission from a state $S_{1}$ to ground state $S_{0}$ of same spin multiplicity is called fluorescence (figure 2). This transition compling with the rule of no change of spin value and therefore has a short lifetime, generally in the nanoseconds range [26]. Alternatively, an electron's transition can be from $S_{1}$ to first triplet state $T_{1}$ by another non-radiative process known as intersystem crossing. Triplet is a quantum state of a system with spin of 1 , with for spin components, of $-1,0$ and +1 [25]. Conversion of $\mathrm{S}_{1}$ to $\mathrm{T}_{1}$ is a spin-forbidden transition as it violates the rule of no change of electron spin. With a longer wavelength shift relative to fluorescence, molecules in $T_{1}$ return to singlet ground state $\mathrm{S}_{0}$ through a radiative process called phosphorescence (figure 2). The probability of Intercrossing system and $T_{1}$ to $S_{1}$ transition are much lower and consequently the lifetime is significantly larger. Molecules comprising heavy atoms such as bromine and iodine are frequently phosphorescent as the heavy atoms facilitate intersystem crossing and thus enhance phosphorescence quantum yields [24].

A range of the environmental factors has a profound influence on fluorescence emission. The first and foremost factor is the solvent polarity that dictates the interactions between the fluorophore and solvent molecules in the local environment. A change in the solvent polarity can cause a spectral shift in emission spectra [27]. Other factors include solvent viscosity, rate of solvent relaxation, rigidity of the local environment and the localized concentration of the fluorescent species [28]. The sensitivity is not similar for all fluorophores. Local environmental factors severely affect the quantum yield as well as the absorption and emission spectra of the fluorophore [29]. In fact, the influence on fluorescence properties is majorly related to the interactions that happen in the surrounding environment during the lifetime of the excited state.

Autofluorescence phenomena follows the three principals, explained below. 


\subsubsection{The Stokes Shift}

In the fluorescence processes, there exists a shift in the emission spectrum with respect to absorption spectrum toward longer wavelengths which is known as Stokes shift. Due to loss of energy during the non-radiative process, the energy associated with emission in lower to that of an absorption energy. The amount of Stokes shift is a measure of the relaxation process happening in the excited state [26].

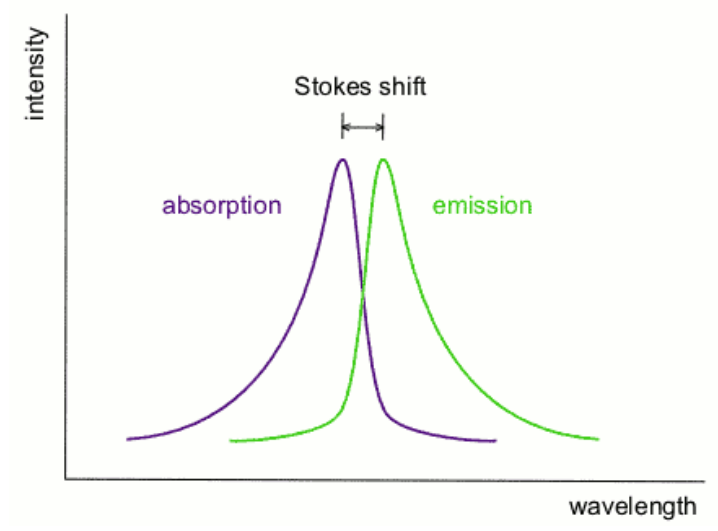

Figure 3: An illustration of an absorption and emission spectrum and Stokes shift

\subsubsection{Kasha`s Rule}

According to Kasha's rule another common feature of fluorescence is that the emission spectrum is independent of absorption spectrum and is identical for each particular fluorophore. Upon excitation into higher vibrational levels, the surplus energy is promptly converted into IR radiation, leaving the fluorophore in the lowest vibrational level of $\mathrm{S}_{1}$ ( occurs in about 10-12 sec) and is presumably a result of a strong overlap among numerous states of nearly equal energy [30].

\subsubsection{Frank Condon`s Principle}

The probability of a transition occurring from the ground state $\mathrm{S}_{0}$ to the excited singlet state $S_{1}$ relies on the degree of resemblance between the vibrational and rotational energy states when an electron exists in the ground state versus those present in the excited state [31] [32]. Upon absorption of a photon of sufficient energy, the molecule undergoes a socalled vertical transition to the excited electronic state. It is known that the proton or 
neutron mass is approximately 1870 times that of an electron and that electrons can move much faster than nuclei. Consequently, as these transitions occur in such a short time interval (femtoseconds) and considering the large inertia of nuclei, electronic motions when observed from the perspective of the nuclear coordinates transpire as if the nucleic were static [24] [33]. The nature of this transition was explained by the Franck-Condon principle that an electronic transition is most probably to happen with no changes to the relative coordinate of the nuclei in the molecular entity and its surroundings. This principle further explains that while nuclear coordinate changes are negligible relative to electronic motions, those electronic transitions are favored that corresponds to a minimal change in nuclear coordinate (figure 4).

Frank-Condon principle applies both to emission and absorption transitions. Return to the ground state usually happens to a higher excited vibrational ground state level, and is followed by reaching to thermal equilibrium promptly. Another characteristic of such kind transitions is that electronic excitations do not significantly revise the nuclear geometry [34]. Therefore, the spacing of the vibrational energy levels of the excited states is analogous to that of the ground state. An interesting consequence of the aforementioned facts along with Kasha's rule states that the emission spectrum is normally a mirror image of absorption spectrum. In Conclusion, electronic transition structure perceived in absorption and emission spectra are identical [35] [36].

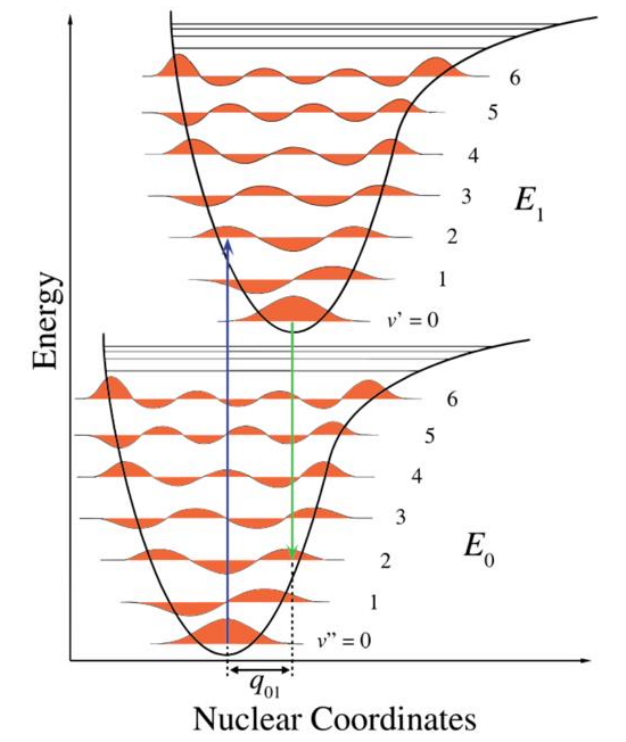

Figure 4: Franck-Condon principle energy diagram 


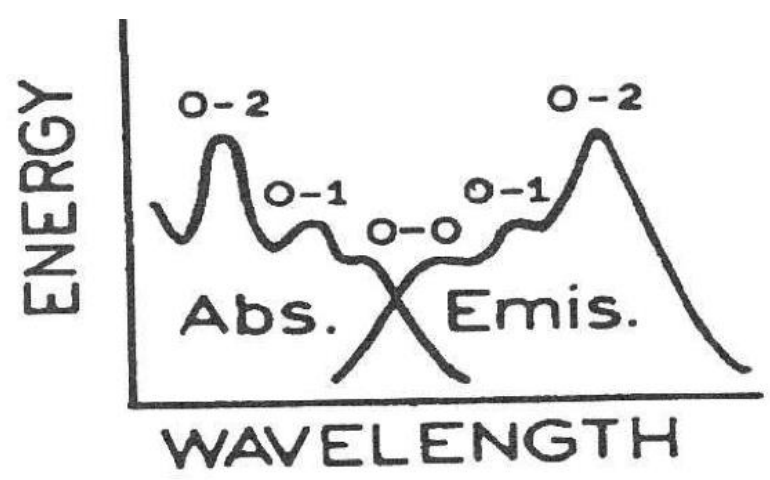

Figure 5: Representation of the absorption and fluorescence spectra corresponding to the energy diagram [37]

\subsection{Autofluorescence Microendoscopy Imaging 1.4.1 Endogenous Sources of Autofluorescence and Origins of Normal- malignant Contrast}

In the development of noninvasive optical guided biopsy, normal tissues can be statistically discriminated from malignant tissues by examining their autofluorescence (AF) spectrum. AF molecules are abundant in normal tissues. Among endogenous fluorophores, NADH, flavins and the extracellular matrix (ECM) are major contributors of AF generation because of their intrinsic properties [38]. According to Kasha's rule, each of these endogenous fluorophores have a known and unique excitation and emission spectra which is shown in figure 6. In breast ducts, the collagenous part of extracellular matrix is the main source of emitted AF and therefore emission light is in the green range [39]. 

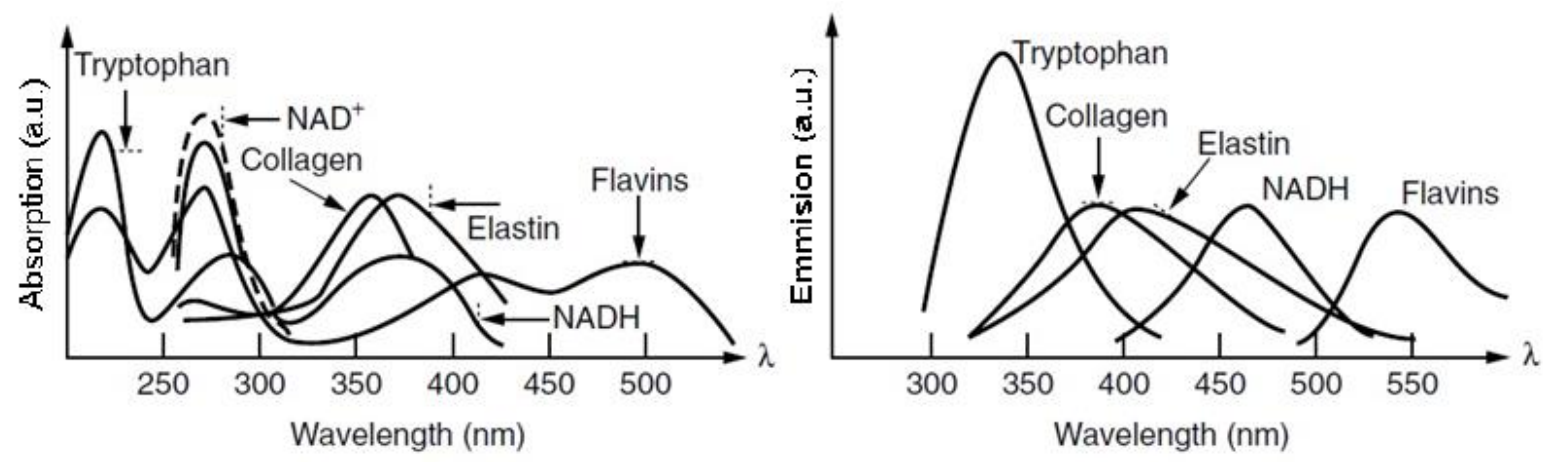

Figure 6: Absorption and emission spectra of important endogenous fluorophores [40].

The perceived cancer-normal contrast in the AF spectrum are indications of biochemical and morphological alterations of endogenous AF sources in tissue upon cancer invasion [40]. The physiological differences between normal and cancer would significantly influence the optical signature of tissue. Firstly, it is known that collagen constitutes the scaffold of tumor microenvironment and affects tumor microenvironment such that it regulates ECM remodeling by collagen degradation, and promotes tumor infiltration, and invasion [41]. Degraded collagen exhibits different optical properties compared to normal collagen, and most importantly lacks the ability of collagen to fluoresce. The decrease in the concentration of fluorophore upon tumor invasion would result in a drop in intensity of AF generated and in turn to normal-malignant contrast in AF imaging (figure 7a) [42]. Secondly, tumor growth would also revise the optical properties of the tissue due to angiogenesis. One of the major requirements for cancer cells to survive and grow is formation of a new network of blood vessels to obtain blood and nutrients to the tumor, which is called angiogenesis [43] [44]. Tumor has significantly more vasculature compared to normal tissues. Total hemoglobin concentration and oxygen saturation of lesions are highly correlated with malignancy [45]. Both oxygenated and deoxygenated hemoglobin possess relatively high light absorption in visible range, hence tumor manifest a drastically higher absorption compared to normal tissue (figure 7b) [28] [46]. Consequently, the possibility of absorption of illumination and emitted AF lights will increase, and that results into a decrease in the intensity of AF. 


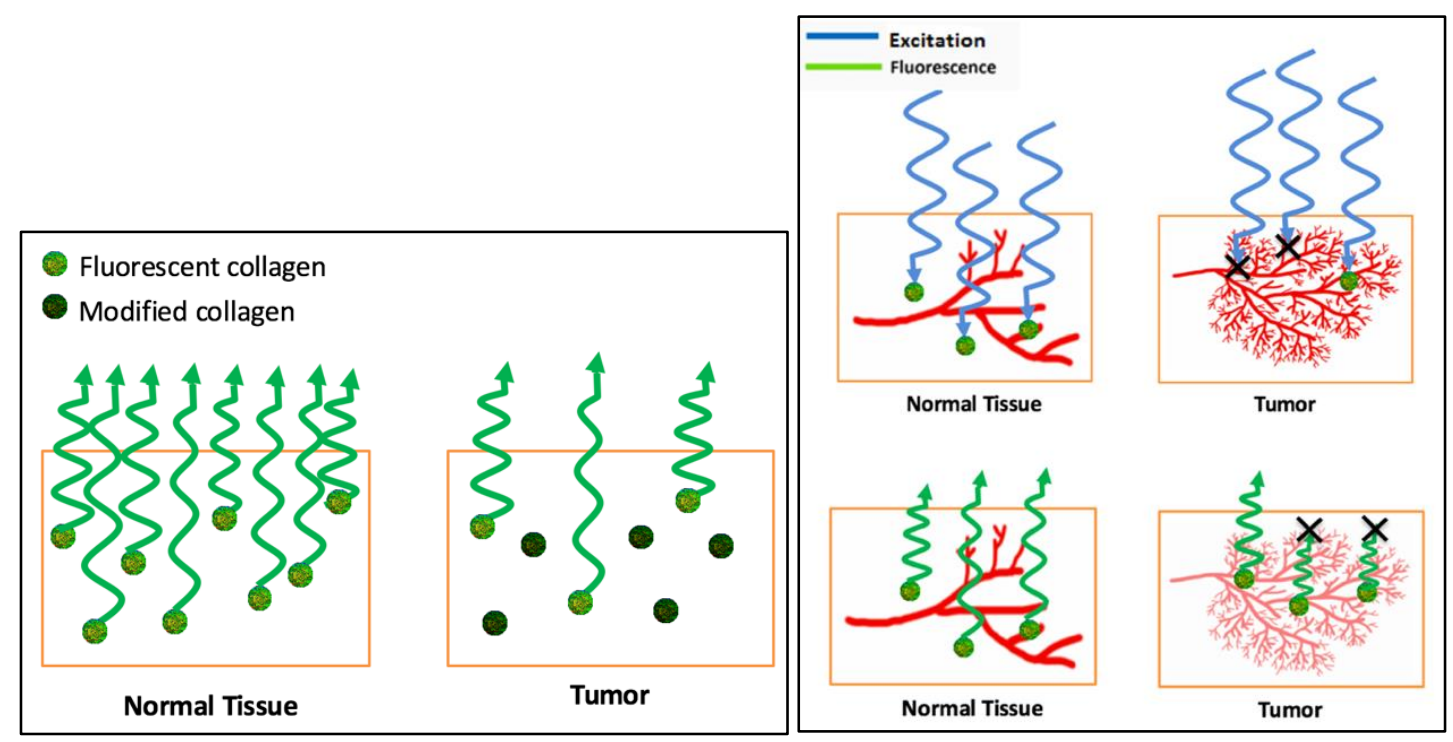

(a)

(b)

Figure 7: Sources of contrast a) An illustration of collagen modification upon cancer growth that reduce the intesity of autofluorescence b) Tumor has highier absorption with respect to healthy and therefore absorbs both excitation and fluorescence lights more

\subsubsection{Autofluorescence Endoscopy}

Previous studies showed that autofluorescence (AF) real time imaging is a feasible surveillance tool for guiding biopsies [47] [48]. Fluorescence guided biopsy is a way of looking directly at the tissue that can ascertain the characteristic of the region of interest. The ultimate aim is to provide the diagnostic radiologist with an additional tool to help differentiate between benign and malignant, thus reducing the sampling error of biopsies. In conventional techniques, biopsies are taken under white light guidance. In colonoscopy suspicion lesions are recognized by their raised structure. However, flat dysplasia is not evident on gross endoscopic inspection and tissue sampling in the screening process is to some extent random [49] [50] [51]. The fact that foci of dysplasia could be very small can lead to large sampling errors [52]. Likewise, in esophagus endoscopy, white light Imaging (WLI) performance in identifying early neoplastic changes is poor since it detects the neoplasia based on superficial vasculature and surface structure changes [53] [54] [55]. 


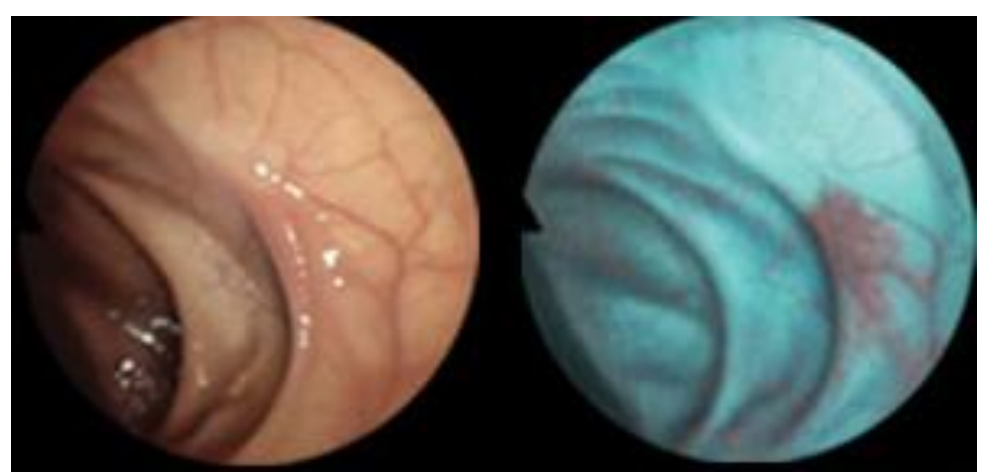

Figure 8: White light(left) and autofluorescence (right) endoscopic images of dysplasia colon. The autofluorescence images shows a clear cancer-malignant contrast [56].

AF imaging distinguishes cancer versus non-cancer based on the spectroscopic characteristics of tissue. While malignancy invasion drastically changes the corresponding characteristics of tissue, AF imaging of colon was capable of revealing malignancy as a different color with respect to normal (figure 8) [56] [57]. Studies on esophageal cancer showed a similar result, while the malignancy in AF imaging was appeared as a shadow (low fluorescence signal) compared to normal tissue and therefore is significantly more visible (figure 9) [58] [59] [60].
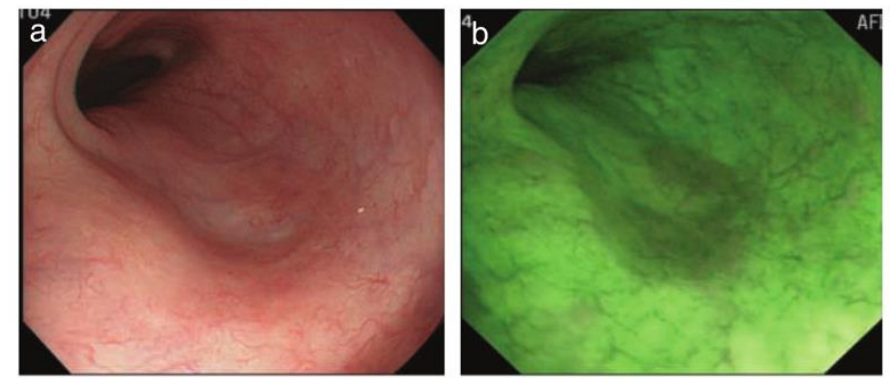

Figure 9: a) White-light imaging of Type Ilc esophageal cancer in the posterior wall of the lower esophagus. (b) On autofluorescence view, the lesion could be located as darker areas [59].

\subsubsection{Shortcomings in Breast Cancer Diagnostic Methods}

As mentioned previously breast cancer may present either as a lump or a suspicions mass in mammograms. Subsequently the diagnosis will be confirmed by ultrasound and biopsies if required. There exist an area of uncertainty known as BIRAD $3^{1}$ in screening mammographic abnormalities which occur in $6 \%$ of examinations and despite their low

\footnotetext{
1 The Breast Imaging Reporting and Data System (BI-RADS), established by the American College of Radiology, offers a standardized classification for mammographic studies. This system explains good correlation with the likelihood of breast malignancy. The category ranges from BIRAD 1, negative findings, to BIRAD 6 Known biopsy-proven malignancy (Eberl, Fox, Edge, Carter, \& Mahoney, 2006).
} 
malignancy rate, they require additional follow-up or even biopsy [61]. Biopsy is the most accurate clinical technique that provides the definitive diagnosis of cancer versus benign tissue. Ultrasound-guided core-needle breast biopsy is an essential method for radiologists who need to sample detected suspicious lesions [62]. Biopsy is unavoidably invasive and highly reliant on the radiologist's skills. Furthermore, biopsies are subject to sampling error due to the limitations of ultrasound to delineate the malignancy (figure 10). Biopsies may need to be repeated in cases where ultrasound interpretation is not in agreement with biopsy results where ultrasound indicates malignancy but pathology results are negative. Not all precancerous cells will develop into cancer and existing imaging modalities are incapable to make the distinction. Unnecessary biopsies could have been avoided if a direct visualization of the abnormality had been possible.

Optical-guided biopsy, as an alternative method to conventional biopsy, provides less invasive and quantifiable possibility for cancer diagnosis. This technique, which engages tissue spectroscopic imaging to biopsy classifies tissues depending on their endogenous components that can be more compatible to pathology results compared to ultrasound interpretation. This method has the potential to improve the safety, sensitivity and specificity of cancer diagnosis and hence reduce the number of repeated biopsies [63] [64].

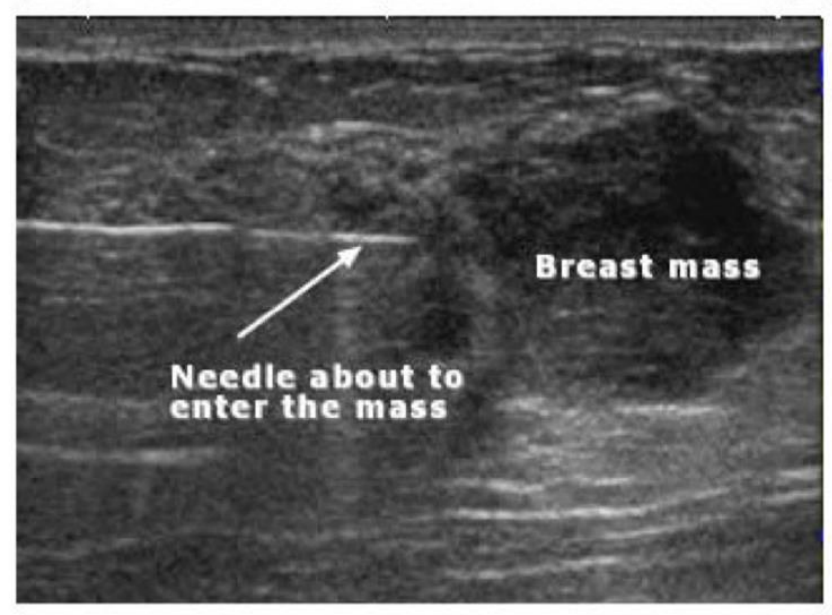

Figure 10: Ultrasound-guided breast biopsy [64]. 


\subsubsection{Ductoscopy vs. Interstitial Microendoscopy}

Ductoscopy is a way of looking directly at the tumor by reaching the suspicious area through breast ducts using a submillimeter endoscope (figure 12). In previous studies, standard white-light ( $6.3 \mathrm{~mW}$ broad-band) was employed for microendoscopic exploration of breast cancer through ducts along with AF imaging (5.3mW blue band excitation (390$450 \mathrm{~nm})$ ) of the tissue of interest recorded at 30 frames/sec. In this study the microendoscope had a diameter of $0.7 \mathrm{~mm}$. Cancer had significantly less AF relative to normal tissue when excited by blue light, so in the false color image normal tissue appears cyan which means presence of AF signal, while abnormal tissue had a range of red color in each pixel, depending on the red-to-green ratio which in false color means absence of AF [6] [7] [8]. A comparison between AF imaging and standard white light microendoscopy depicted a better contrast in the former. While malignancy appeared as lumps under white light imaging a distinctive color boundary between normal and cancerous tissues were observed with AF microendocopy (figure 11) [65]. Nevertheless, ductoscopy has limited practical applications in the diagnostic and management of breast cancer. Occasionally, the AM did inadvertently pierce the duct wall into the surrounding breast tissue. AF images were still generated that differentiated cancer from normal tissue, although the variance was less obvious. This observation lead to proposing an innovative use for the AM with significant potential clinical applications if applied interstitially and coupled with diagnostic imaging and biopsy (figure 12). Interstitial AF microendoscope can investigate the area under study through the same interstitial channel created by the trocar needle to acquire breast cancer biopsies. If the interstitial microendoscopy proves to be effective, this imaging modality can guide breast cancer biopsies. 


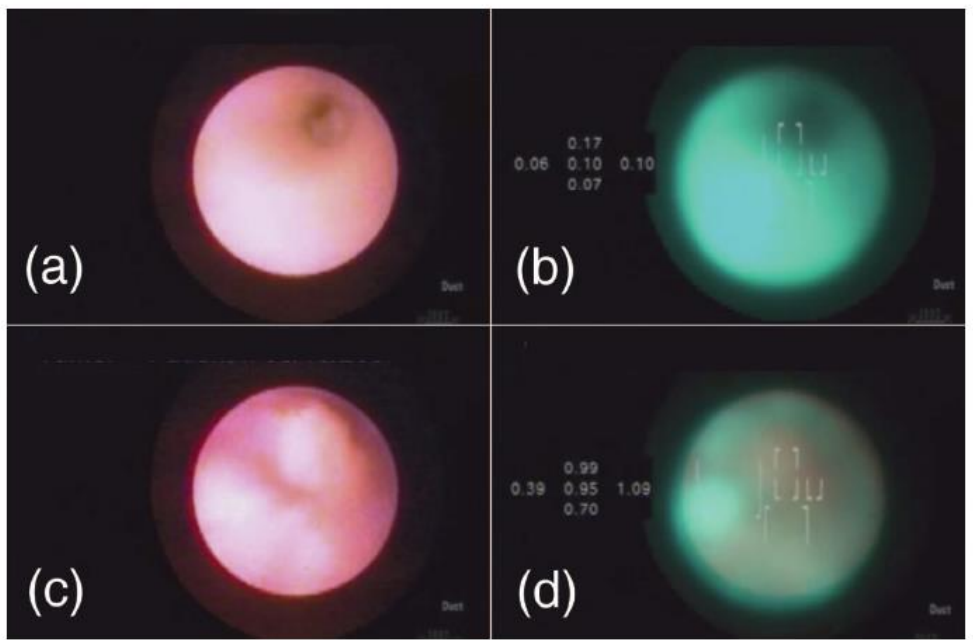

Figure 11: Comparative presentation of normal region[ $a$ white light and b autofluorescence] and tumor region [c white light and d autofluorescence] [6] .
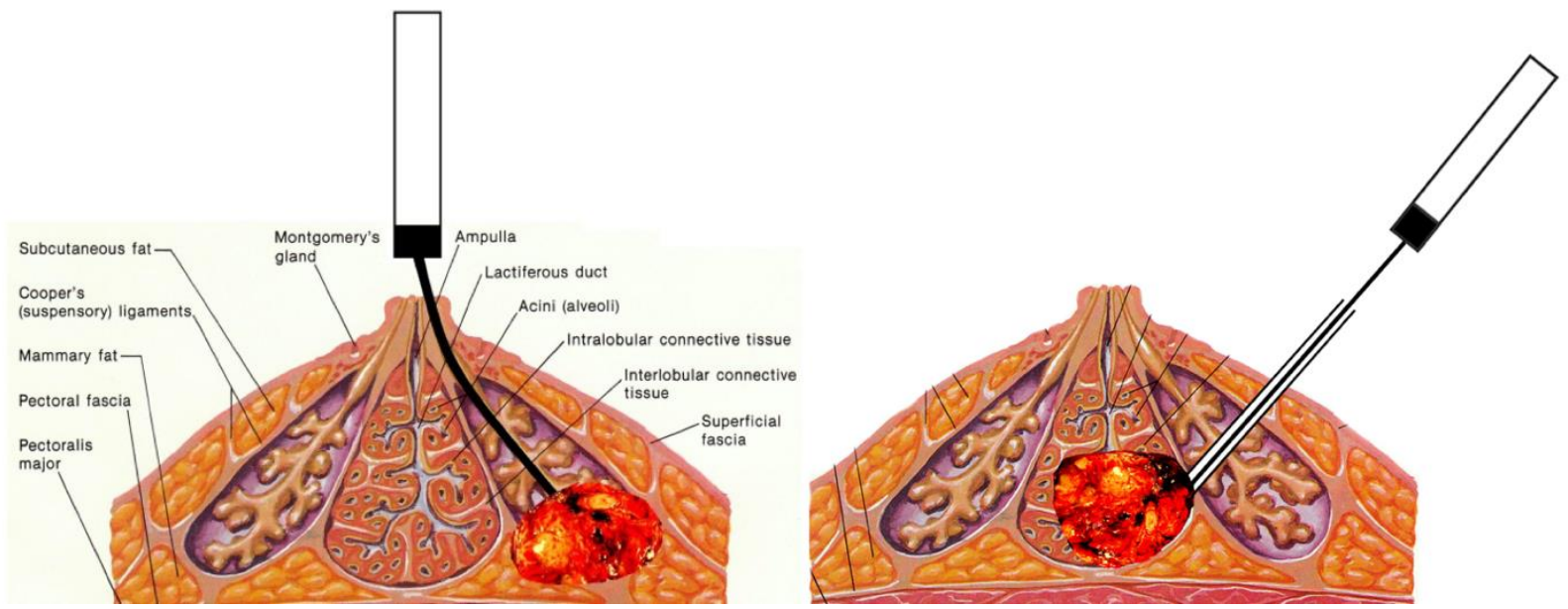

Figure 12: Schematic representation of autofluorescence ductoscopy (left) and Interstitial autofluorescence approach (right

\subsection{Hypothesis and Specific Objectives}

The hypothesis in this study is that the interstitial AM is capable to distinguish between cancer and normal in ex-vivo female tissue. Our main objective is to study the feasibility of autofluorescence microendoscopy (AM) to distinguish between cancers and normal tissue by inserting the AM interstitially into breast tissue of ex-vivo female breast into areas of known cancer and normal tissue within the same breast. Subsequently the AF 
images of both cancer and normal tissues are correlated to biopsy results of samples taking from the same area. 


\section{Chapter 2: Materials and Methods}

\subsection{Patient Recruitment}

The study protocol was approved by the ethics committee at University Health Network (14-8510-CE) and Ryerson University (REB 2015-337). From April 2016 to June 2016, five consented patients were recruited among females diagnosed with clinically palpable breast lumps who are planned to have curative mastectomy at Princess Margaret Hospital (figure 13). Patients diagnosis was confirmed by biopsy and this study had no exclusion criteria. For patients requiring lymph node assessment, the operation was done in the absence of Sulphan Blue dye, as spectroscopic analysis of patent blue dye revealed that this mapping dye would interfere with $\mathrm{AF}$ emitted light and would alter the autofluorescence images. Patient's age, menopause status and histopathology assessment results of samples were included in collected data.

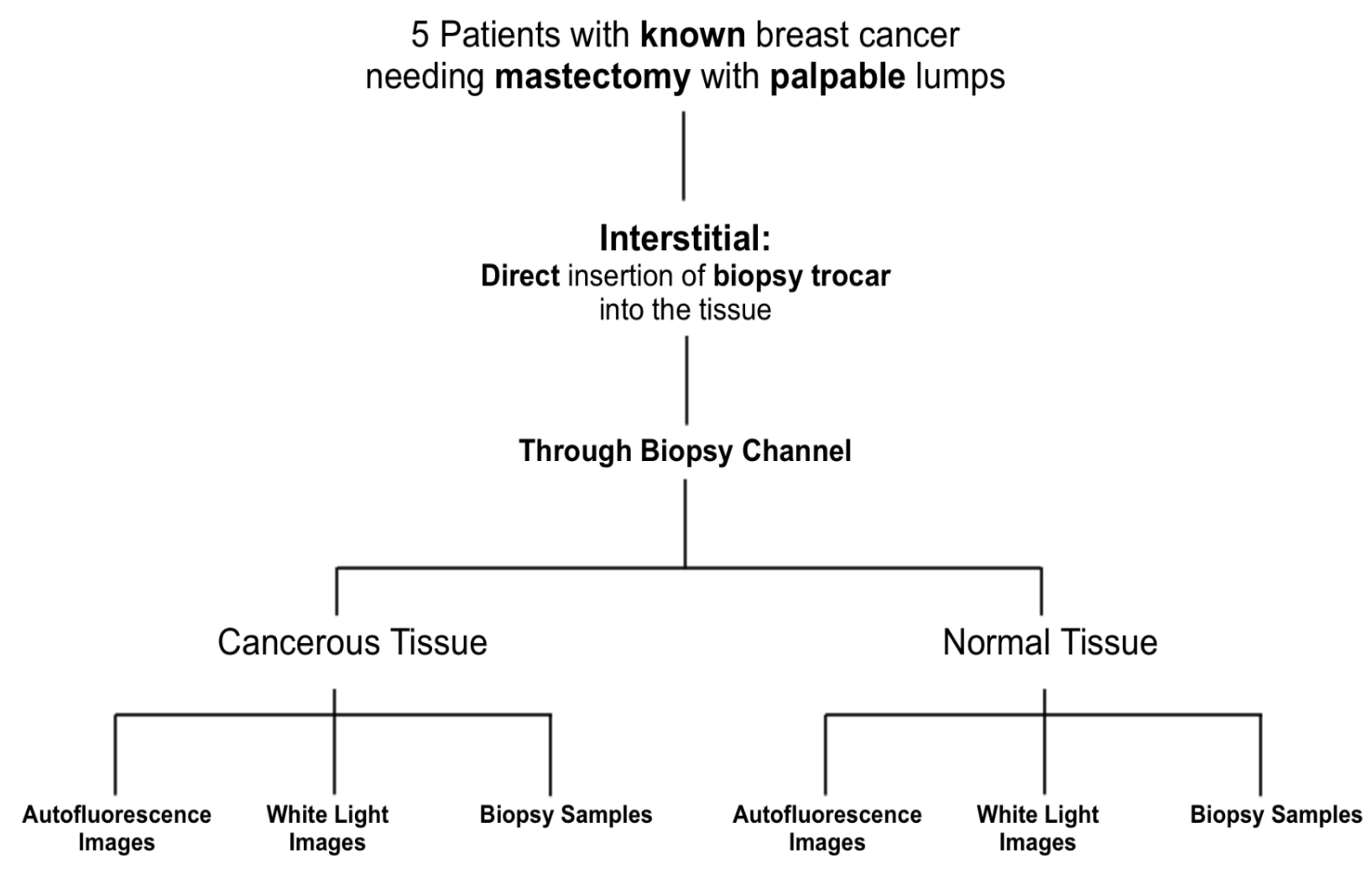

Figure 13: Study Schema 


\subsection{Instrument Description}

Flexible fine scopes composed of fiber bundles are capable of deep imaging in tissue. The fluorescence microendoscope system consisted of a light source, a fiber bundle, and a combination of lenses and a CCD camera connected to a camera controller (figure 12). The illumination light sources were $150 \mathrm{~W}$ short arc super-high-pressure mercury lamps (OncoLIFE $®$, Xillix Technologies Corporation, British Columbia, Canada). This setup operated in two different modes of white light and autofluorescence(AF). Illumination in standard white-light and AF were $6.3-\mathrm{mW}$ broadband light range and 5.3- $\mathrm{mW}$ blue band 390 to $450 \mathrm{~nm}$, respectively. In conventional white light mode, illumination was a standard white light. However, in AF mode, a red light within the wavelength of 690 to $750 \mathrm{~nm}$ was illuminated making the illumination in fluorescent mode purple which is a mixture of blue and red lights. This device can work in both modes of white light and AF, by switching the illumination filters and the corresponding $\mathrm{CCD}$ cameras to each mode.

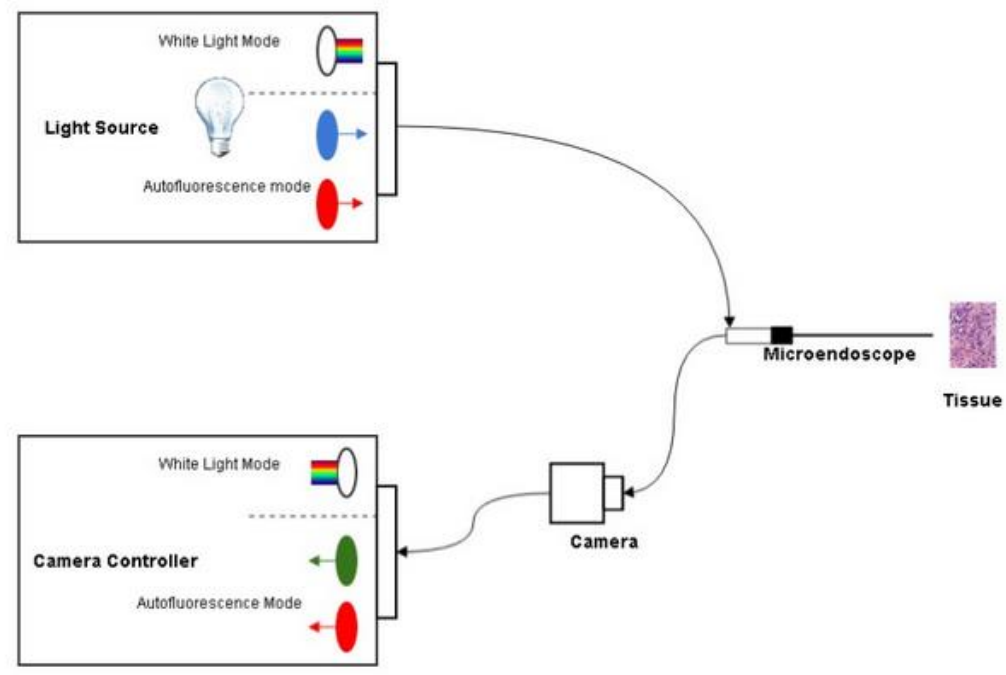

Figure 14: Schematic representation of setup

In $\mathrm{AF}$ mode after the excitation light exits the distal end of the microendoscope to illuminate the tissue, the emitted light is gathered by the collection fibers collated in the center of the probe (figure 15) and guided through an objective and an emission filter prior to delivering the image onto the iCCD camera (OncoLIFE®, Xillix Technologies 
Corporation, British Columbia, Canada). The microendoscope (Polydiagnost, Germany) is semi-rigid and the imaging part was constructed as a 6000 fiber bundle. This device had a working length of $70 \mathrm{~mm}$, field of view of 70 degrees, and depth of view of 1 to 10 $\mathrm{mm}$. Resolution analysis of AM in both AF and white light mode using USADF target 1951 revealed that the microendoscope has a resolution of 10.1 and 4.49 line-pair/mm in white light and AF mode respectively (figure 16).

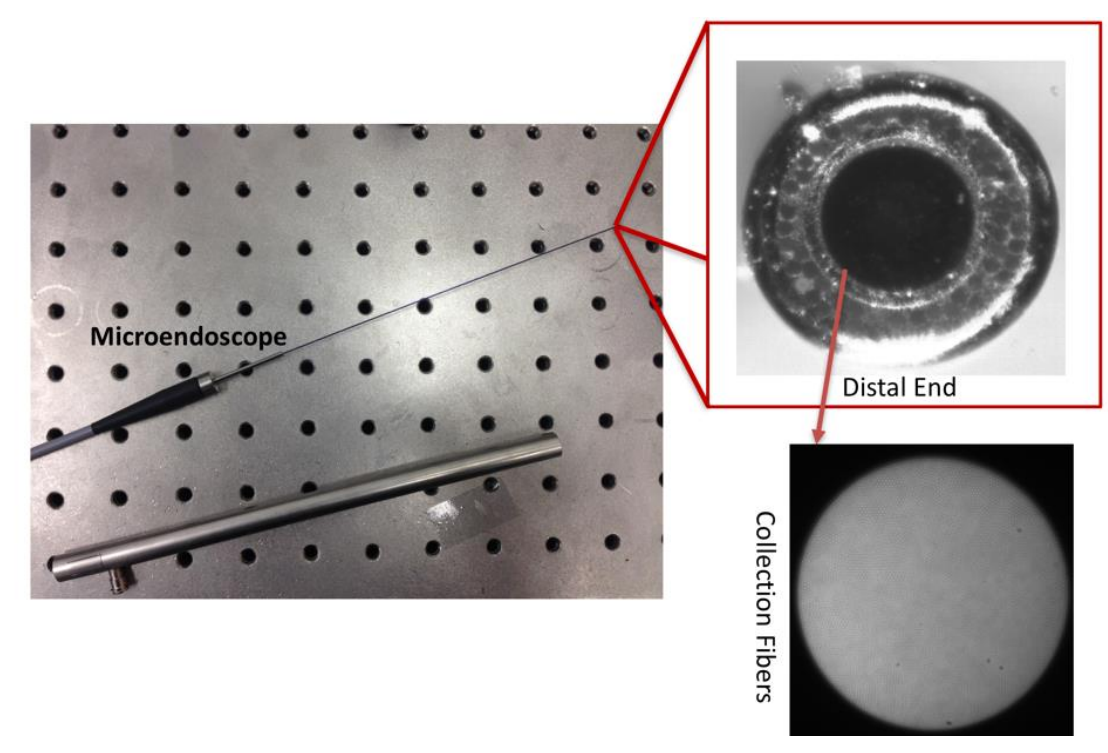

Figure 15: Microendoscope
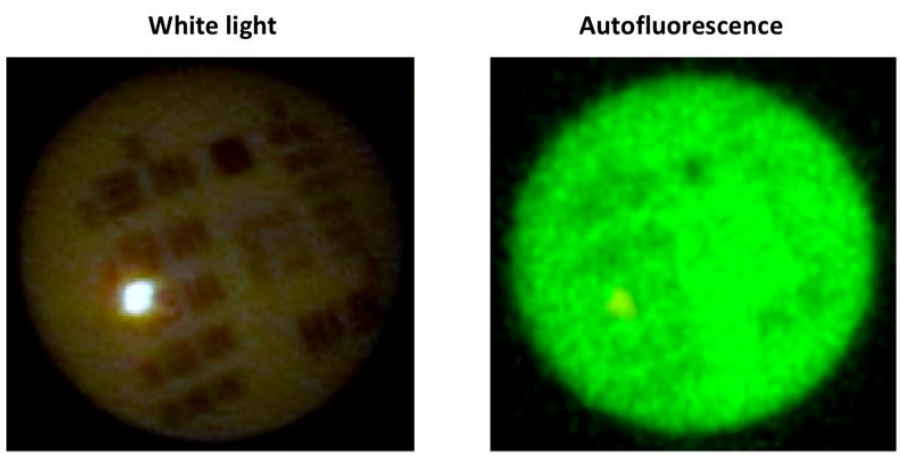

Figure 16: White light (left) and autofluorescence (right) images of USADF 1951 target

As shown in Fig. 14, the microendoscope was coupled via camera to a camera controller imaging system (OncoLIFE®, Xillix Technologies Corporation, British Columbia, Canada). The camera consists of two cameras: a standard three-color CCD for conventional white light and an iCCD for the fluorescence. However, in the fluorescence 
mode both iCCD and three-color CCD are engaged. The iCCD provides the signal to the green channel and three-color CCD provides the signal to the red channel 690 to 750 .

\subsection{Image Formation}

In autofluorescence (AF) mode, the light source emits lights in two distinct wavelength ranges of 390 to $450 \mathrm{~nm}$ (blue band) and 690 to $750 \mathrm{~nm}$ (red band). The blue light can undergo two pathways (figure 17). It can either diffuse reflected or excite endogenous fluorophores in breast tissue (namely, collagen) which in turn will lead to generation of green AF. In contrary red light can only undergo diffuse reflectance (figure 17).

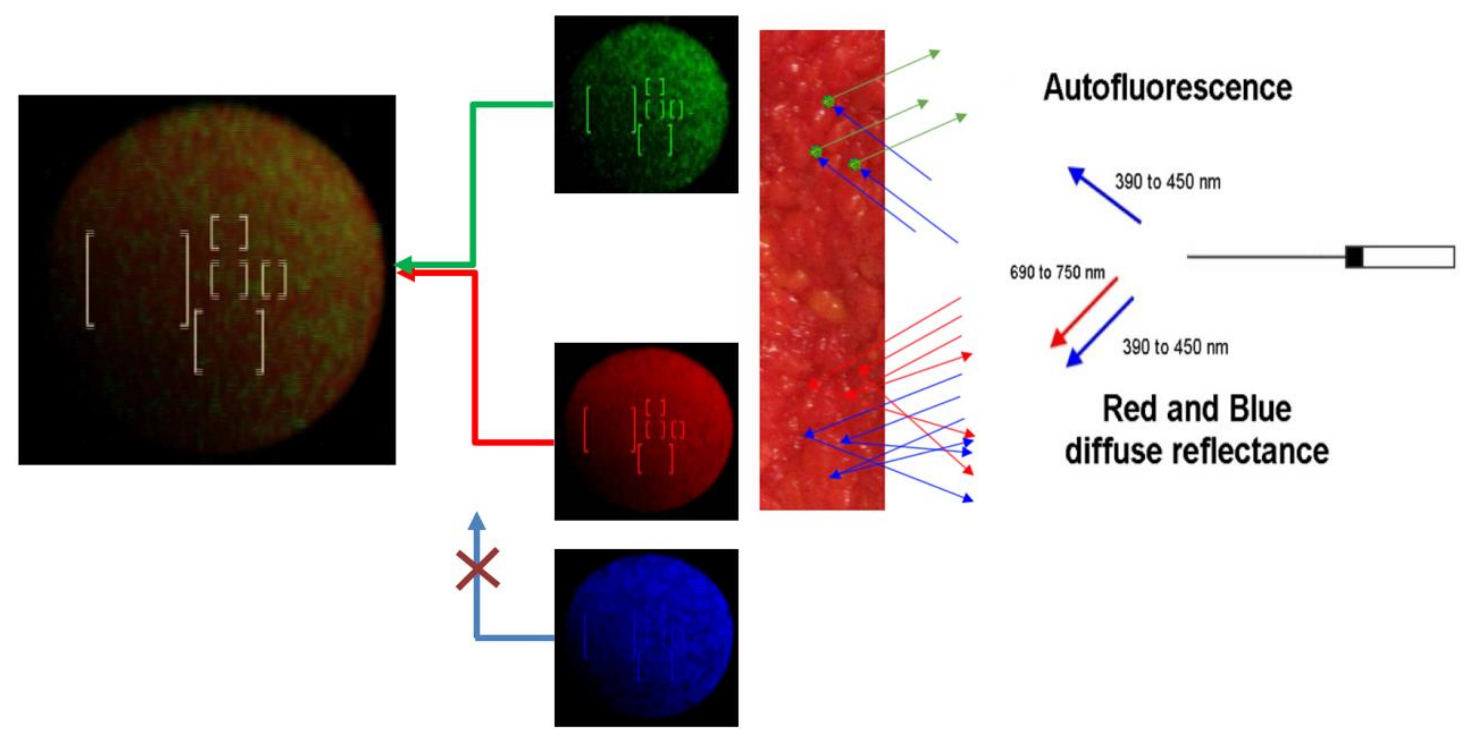

Figure 17: Blue and red illumination pathways and autofluorescence image formation

An autofluorescence image was formed from two distinct images: green autofluoresence and red diffuse reflectance. Overall, malignant tissues relative to normal tissues generate dramatically lesser autofluorescence upon excitation by blue light as described in section 1.4.1. Hence in the false color image the normal tissues should appear as green and cancer as the range of red color, associated with the reduced green $\mathrm{AF}$ and depending on the red to green ratio. Camera controller in AF mode continuously displays five numerical color values (NCV) associated to five areas of interest (indicated by square brackets) and is approximately the ratio of red to green channel intensity averaged over four frames for the central 16*12 pixels (figure 18). Higher NCV values is 
an indication of cancerous tissues and can be used to confirm the abnormality in autofluorescence images.

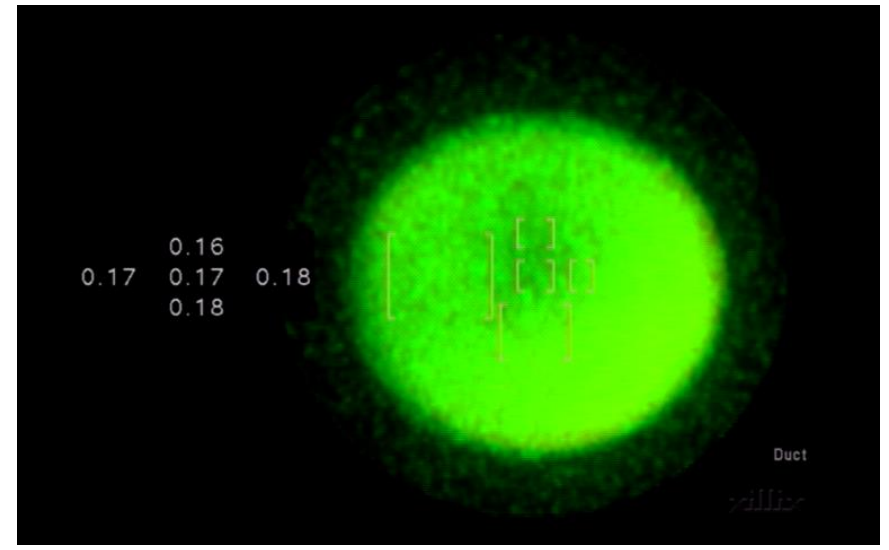

Figure 18: Autofluorescence image acquired from normal fresh pork fat specimen.

\subsection{Autofluorescence Data Analysis}

\subsubsection{Material}

Prior to conducting the real experiment on excised female breasts, AF microendoscopy was tested on random samples that to some extent mimics the properties of the human breast toward developing a feasible operational procedure. Firstly, pork fat was imaged by creating an interstitial channel using a straw. Green autofluorescence images were formed from fresh pork fat (figure 19). Pork meat was drained out of blood. To more precisely mimic the real experiment fresh lamb liver that contains a substantial amount of blood similar to a fresh excised breast was AF imaged. Successfully a red AF image from blood abundant areas was captured (figure 20).

\subsubsection{Image Processing}

The videos were captured by camera controller and later on were converted to digitized data using Capit video converter (MyGica, Shenzhen, China). For the purpose of image processing an open source software called virtualdub (version 1.10.4) was utilized. VirtualDub is a video capture/processing utility for 32-bit and 64-bit Windows platforms licensed under the GNU General Public License (GPL). The software has the feature to 
record AVI raw videos in $\mathrm{YCbCr}$ format. Raw, uncompressed videos were captured in AVI format. Furthermore, to enhance the image analysis, videos were imported to Matlab and converted to RGB format using the equations below. Frames of interest were extracted and averaged over 4 to 12 frames to form a still image. In order to study the autofluorescence data, images were dechannelized to red and green channels. Channels were plotted on a same graph to make the comparison possible as depicted in figure 19 .

$$
\begin{gathered}
R=Y+1.402 .\left(C_{R}-128\right) \\
G=Y-0.34414 .\left(C_{B}-128\right)-0.71414 .\left(C_{R}-128\right) \\
B=Y+1.772 .\left(C_{B}-128\right)
\end{gathered}
$$

\section{$Y$ : Luminace component}

$C_{R}:$ Red - difference chroma component

$C_{B}$ : Blue - difference chroma component

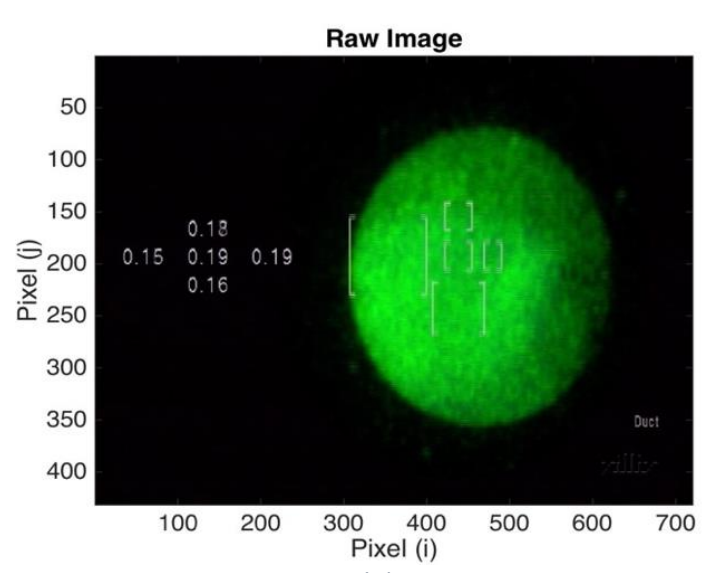

(a)

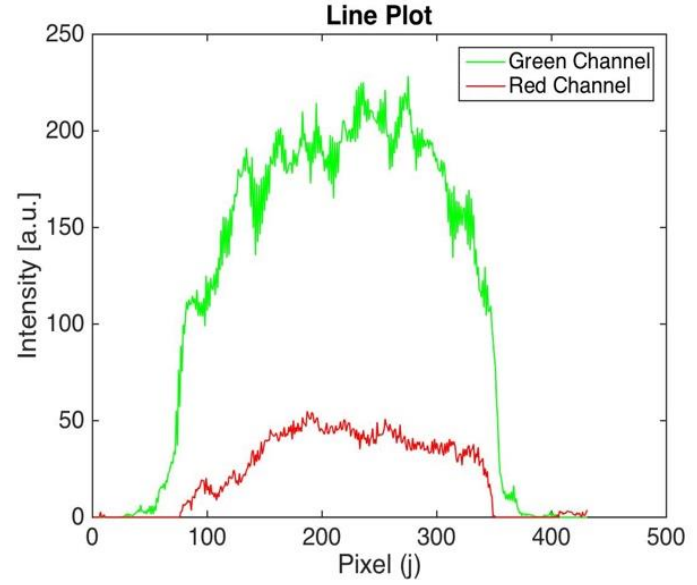

(b)

Figure 19: Autofluorescence image of fresh lamb liver (a) - Line plot of $R, G$ and $B$ channels along the center of the image ( $i=450$ ) (b) 


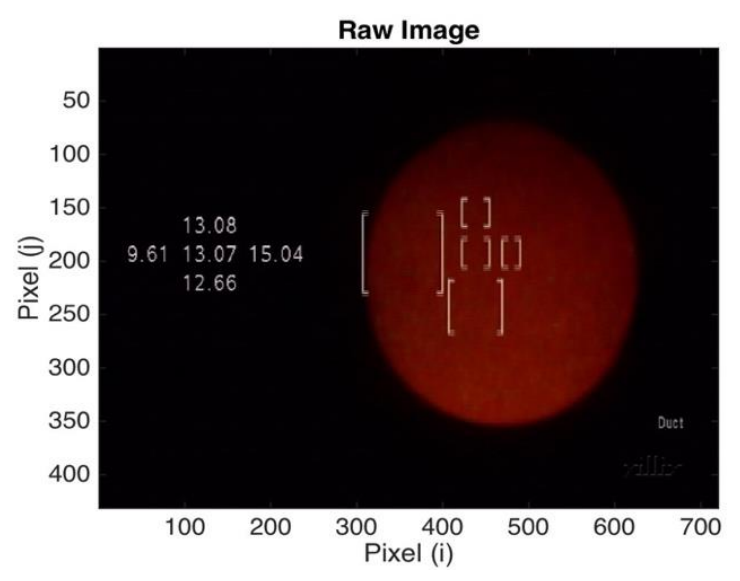

(a)

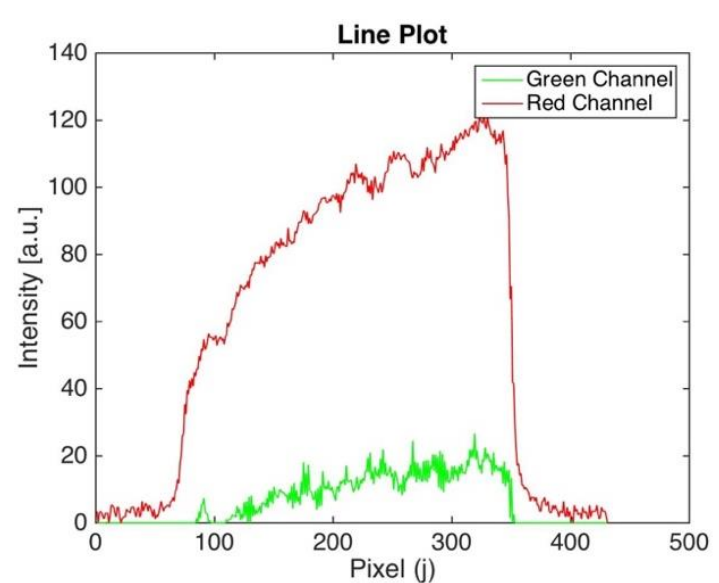

(b)

Figure 20: Autofluorescence image of fresh lamb liver containing relatively larger amount of blood (a) - Line plot of $R, G$ and $B$ channels along the center of the image(b)

\subsection{Standard Operational Procedure}

\subsubsection{Required Equipment}

1) Micro-endoscope

2) Optic Shifter

3) Short Handle

4) Polyshaft

5) Ocular

6) Optic protective tube

7) Luerlock

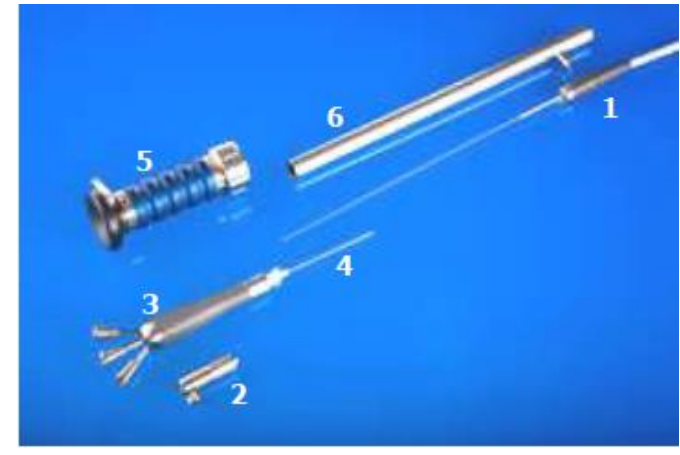

Figure 21: Required Equipment- items 1-6

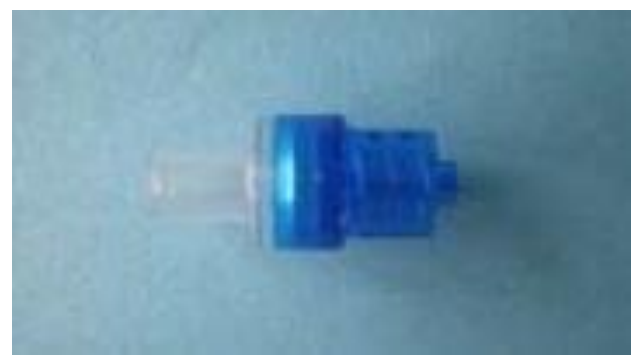

Figure 22: Required Equipment - LuerLock
8) Luerlock Cap
9) Modified Trocar 


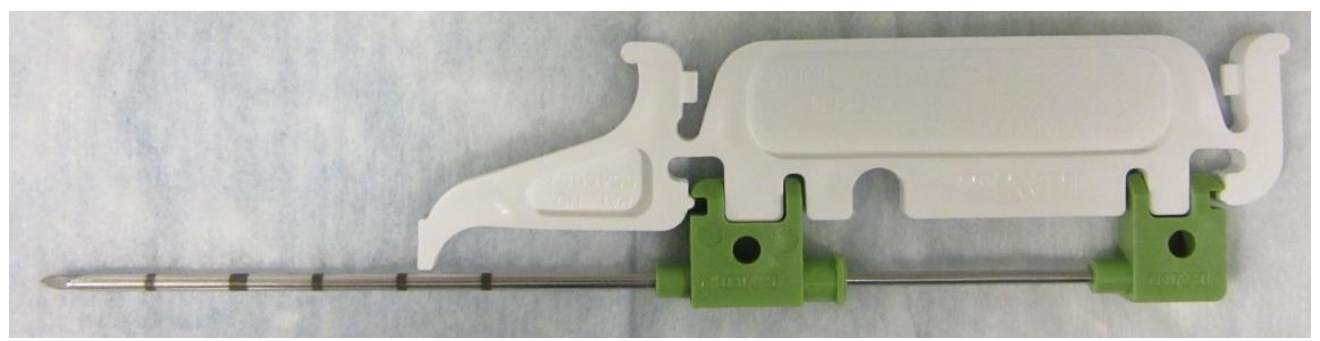

Figure 23: Required equipment-modified trocar

1) Sterile normal saline and 5 cc Syringe

2) BARD magnum biopsy gun: attaches to trocar to obtain tissue biopsy

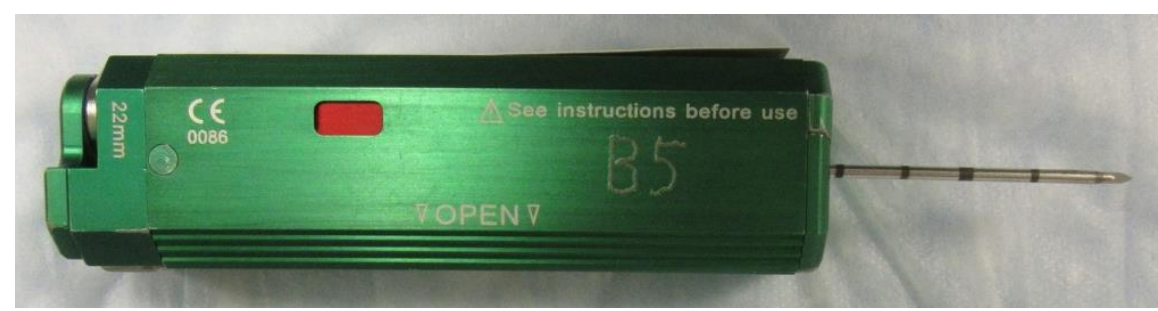

3) Figure 24: Required Equipment- Magnum gun for acquiring biopsies

\subsubsection{Setup preparation}

1) As shown in the picture below the short handle consists of three ports.

Two were used in this experiment:

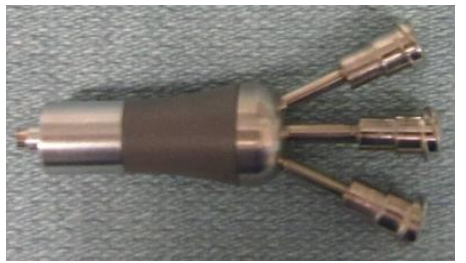

Figure 25: Required Equipment - Short Handle

2) The polyshaft was attached to the short handle.

3) On the short handle the first port was blocked by the Luerlock cap to prevent the egress of saline solution from the unused port. The microendoscope was passed down the optic shifter and connected to the middle port (figure 26). In order to prevent damage to the delicate probe, the tip of the microendoscope was aligned with the distal end of the Polyshaft and Microendoscope was secured by 
the optic shifter screw. Lastly, a syringe was filled with saline and attached to the third port of Luerlock as illustrated in figure below.

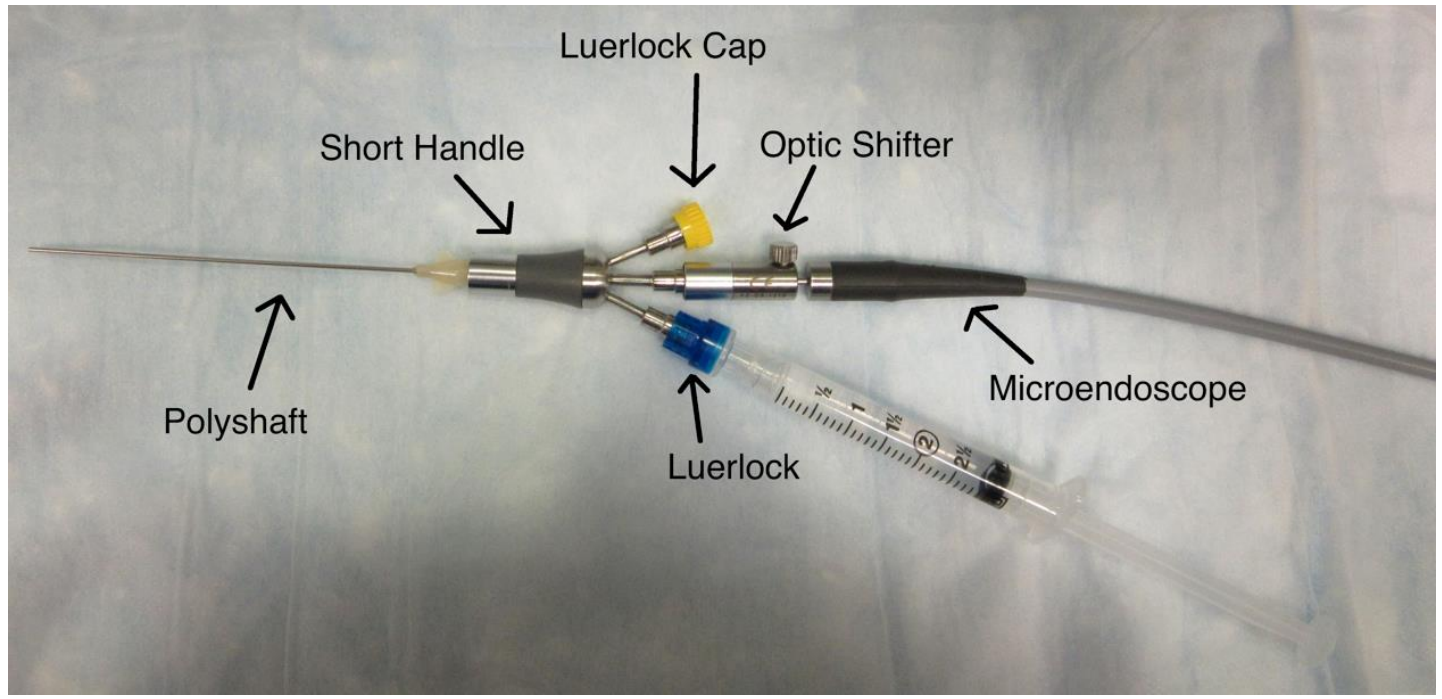

Figure 26: Required Equipment- Data Acquisition setup

\subsubsection{Ex-vivo Procedure}

At Princess Margaret Hospital, succeeding the mastectomy operation, the excised breast was transferred to the pathology lab for histopathology assessment preparation. The policy is that the tissue should be submerged in formalin within a maximum of one hour after the excision. This experiment was conducted in the same lab prior to pathology assessments and all mastectomy specimens were assessed within 20 minutes following the mastectomy excision.

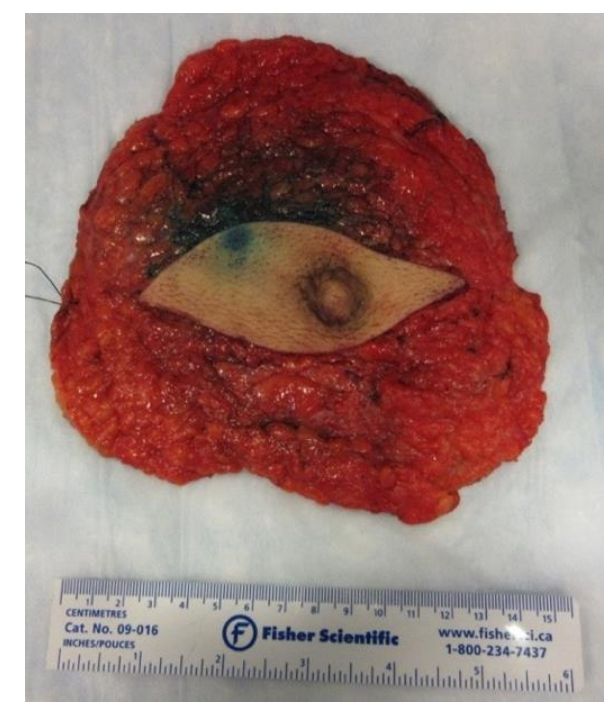

Figure 27: Procedure- Mastectomy specimen 
The experiment was commenced by inserting the trocar into the tumor. Among the eligibility criteria for this study was the palpability of the tumor so that localization of lump was possible. Therefore, for all mastectomy specimens, lumps were successfully located by palpation and in the absence of ultrasound.

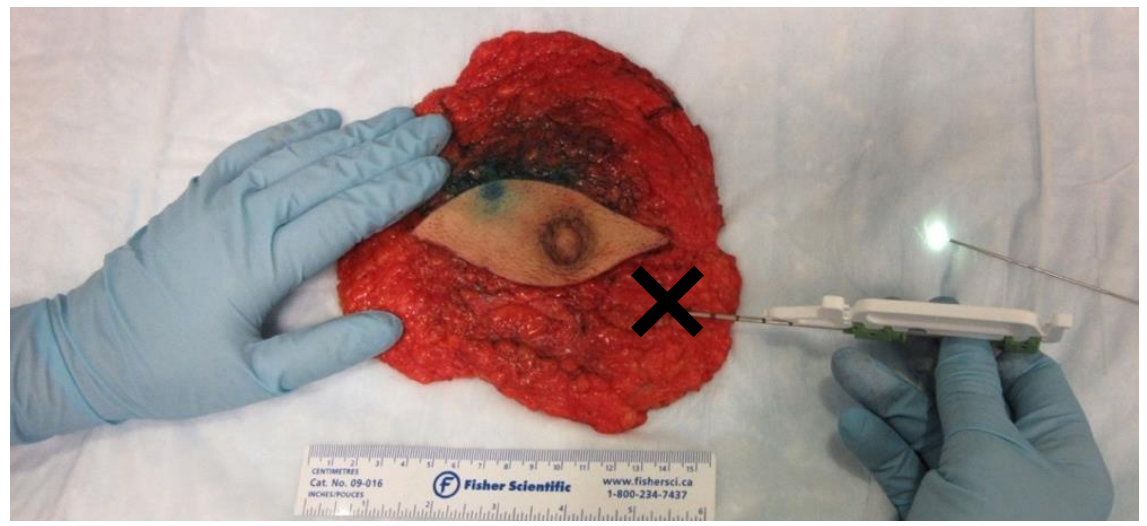

Figure 28: Procedure- Trocar Insertion. Cross signifies the location of tumor.

Next, the stylet inside the trocar was removed. As a result, a clear channel toward the area of interest inside the tumor was created.

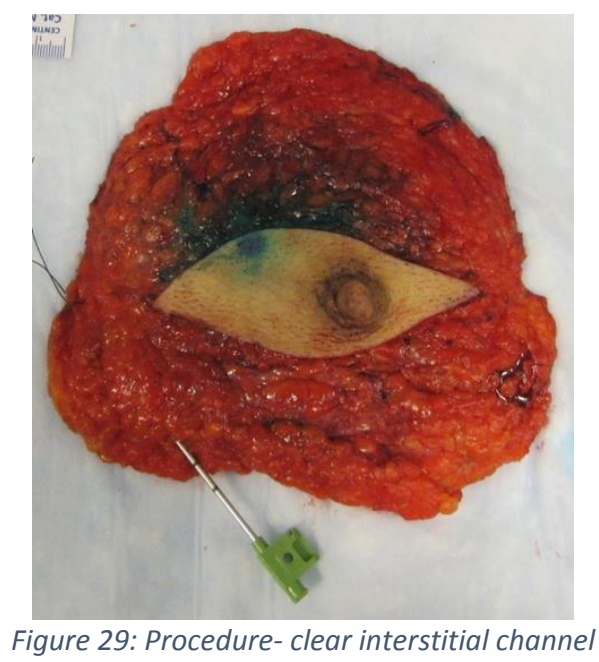

The hollow needle accommodated the data acquisition setup consist of endoscope as shown in figure below. In some cases, the tip of the needle was filled up with blood due to hemorrhage of the fresh tissues. Upon saline rinsing the blood was flushed out from the area and then blood free clear images were captured. Both autofluorescence and white light real-time imaging was acquired. Saline was injected from the saline port, entered the tissue from the Polyshaft and leaked out of the porous tissue or the opening of trocar depending on the 
thickness and firmness of the breast specimen. This method was effective for all cases.

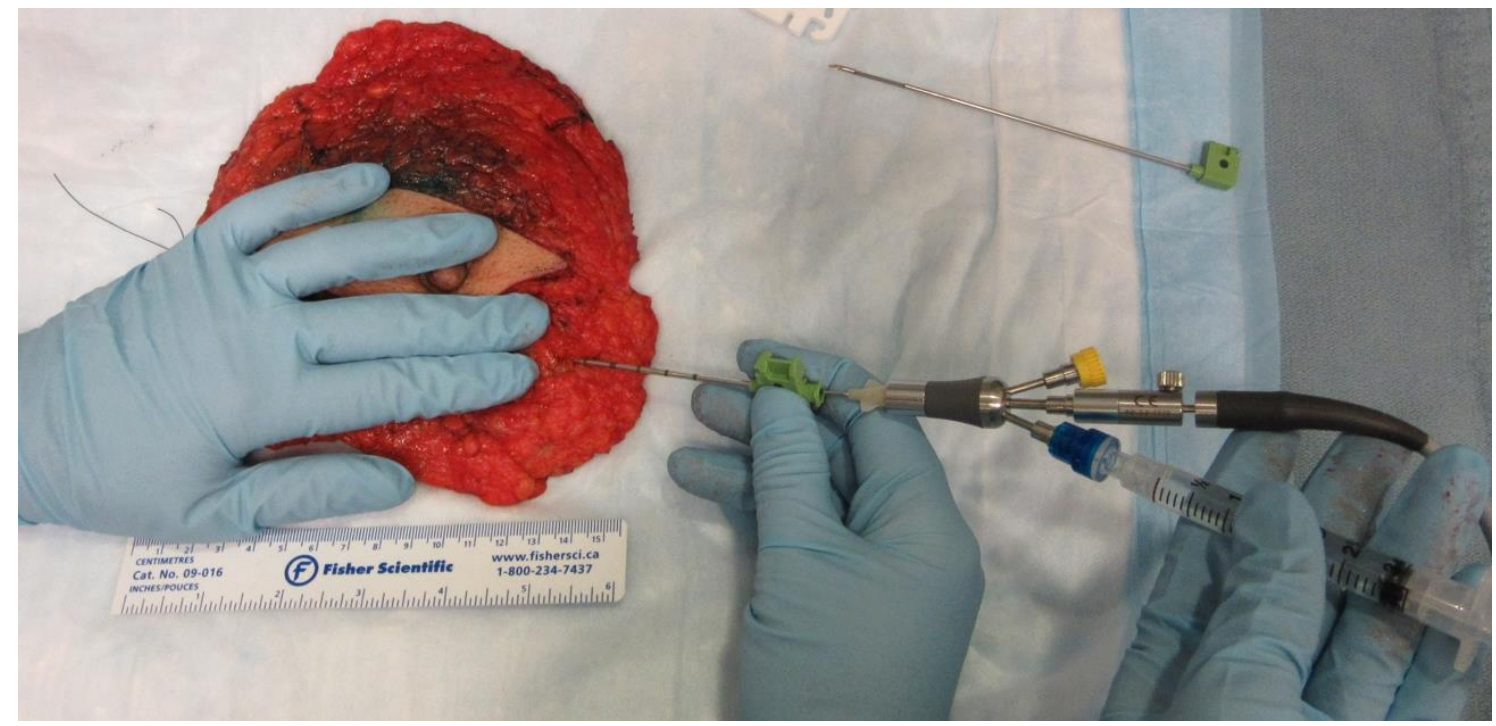

Figure 30: Procedure- Saline injection and Data Acquisition

For breast cancer biopsies in radiology, the exact same trocar is being readily used. A BARD magnum gun (Bard Biopsy Systems, USA) and the insertion trocar was utilized to take core-needle biopsies from the area of interest. After data acquisition, the setup was removed, stylet was inserted into the trocar back to its initial position. The trocar was attached to the biopsy gun and samples from the area under study were taken (figure 31 $\& 32)$.

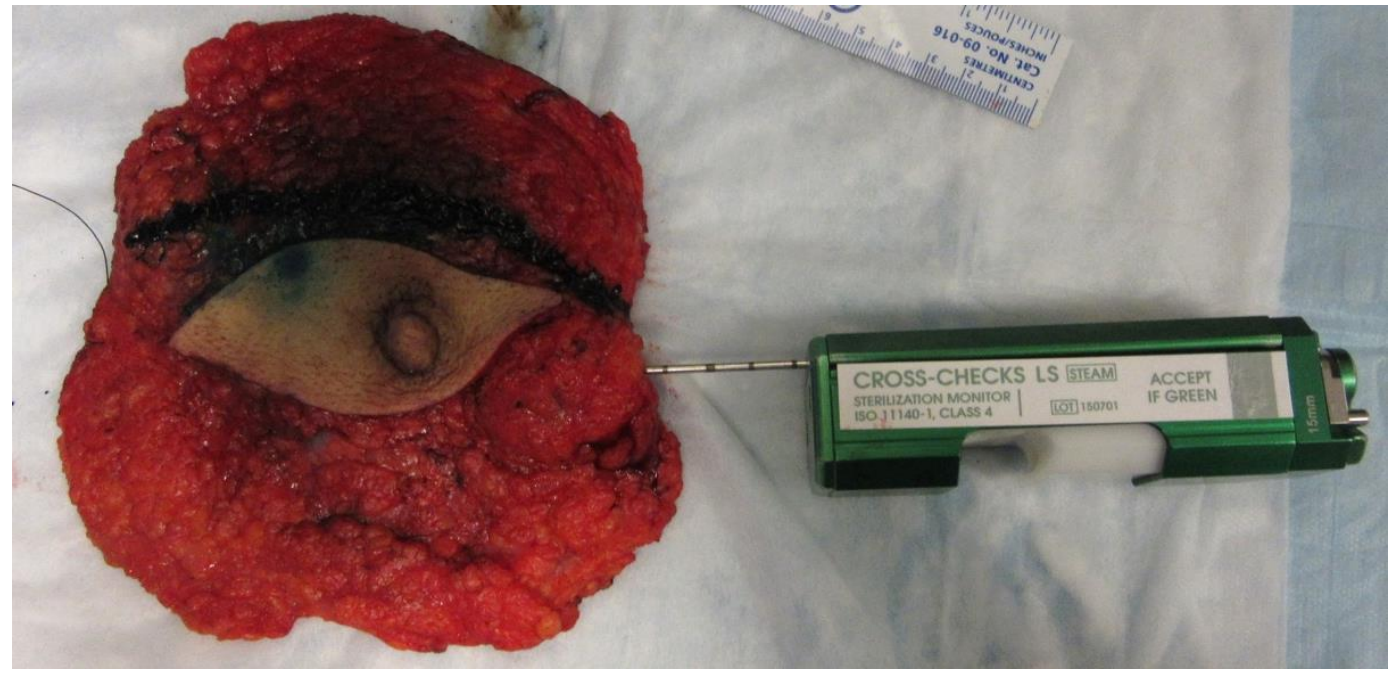

Figure 31: Procedure- Acquiring biopsy samples 


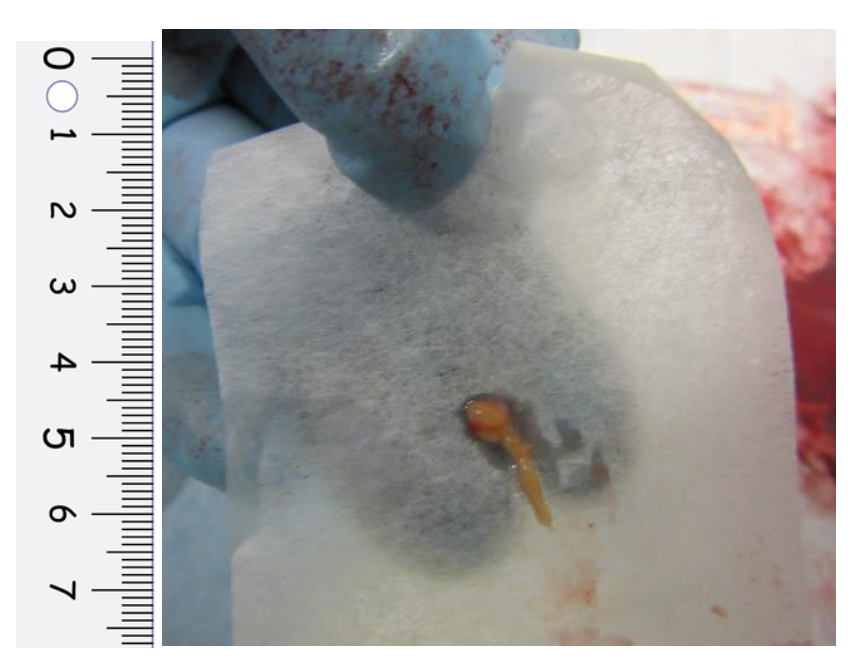

Figure 32: Procedure- Core-needle Biopsy Sample

Biopsy samples were promptly placed into formalin and were sent to pathology for fixation on a paraffin slide. Subsequently H\&E staining was provided to prepare the samples for pathology analysis. The same procedure was repeated for normal areas. Samples from both suspicious and normal tissues were analyzed by pathology assessment. Normal tissues were used as control group. 


\section{Chapter 3: Results}

Table 1: Participants Information

\begin{tabular}{|c|c|c|c|c|c|}
\hline & \multicolumn{5}{|c|}{ Participant No. } \\
\cline { 2 - 6 } & $\mathbf{1}$ & $\mathbf{2}$ & $\mathbf{3}$ & $\mathbf{4}$ & $\mathbf{5}$ \\
\hline Age & 33 & 62 & 43 & 45 & 52 \\
\hline Menoupause Status & Pre & Post & Pre & Pre & Post \\
\hline \multirow{3}{*}{ Hormone Receptor } & ER- & ER+ & ER+ & ER+ & ER- \\
\cline { 2 - 6 } & PR- & PR- & PR+ & PR- & PR+ \\
\cline { 2 - 6 } & HER2- & HER2- & HER2+ & HER2- & HER2+ \\
\hline Grade & III & III & II & III & II \\
\hline Type of Cancer & IDC & IDC & IDC & IDC & IDC \\
\hline $\begin{array}{c}\text { Neo-adjuvant } \\
\text { Chemotherapy }\end{array}$ & Yes & No & No & \multirow{2}{*}{ No } & \multirow{2}{*}{ No } \\
\hline
\end{tabular}

Estrogen receptors (ER) and progesterone receptors (PR; also called $\mathrm{PgR}$ ) may be found in breast cancer cells. Cancer cells with these receptors rely on estrogen or progesterone, to grow [67]. HER2 is the more common name for the gene called ERBB2. The job of HER2 is to control a protein on the surface of cells that helps them grow. Learning if a tumor has estrogen and/or progesterone receptors or HER2 aids doctors decide a patient's risk of recurrence and whether the cancer can be treated with hormone therapy [67]. 


\subsection{Participant \#1}

\section{Pathology results of the entire tumor and lymph nodes:}

- $1.4 \mathrm{~mm}$ tumor $+3.5 \mathrm{~cm}$ mostly fibrosis tissue

- both lymph nodes were negative

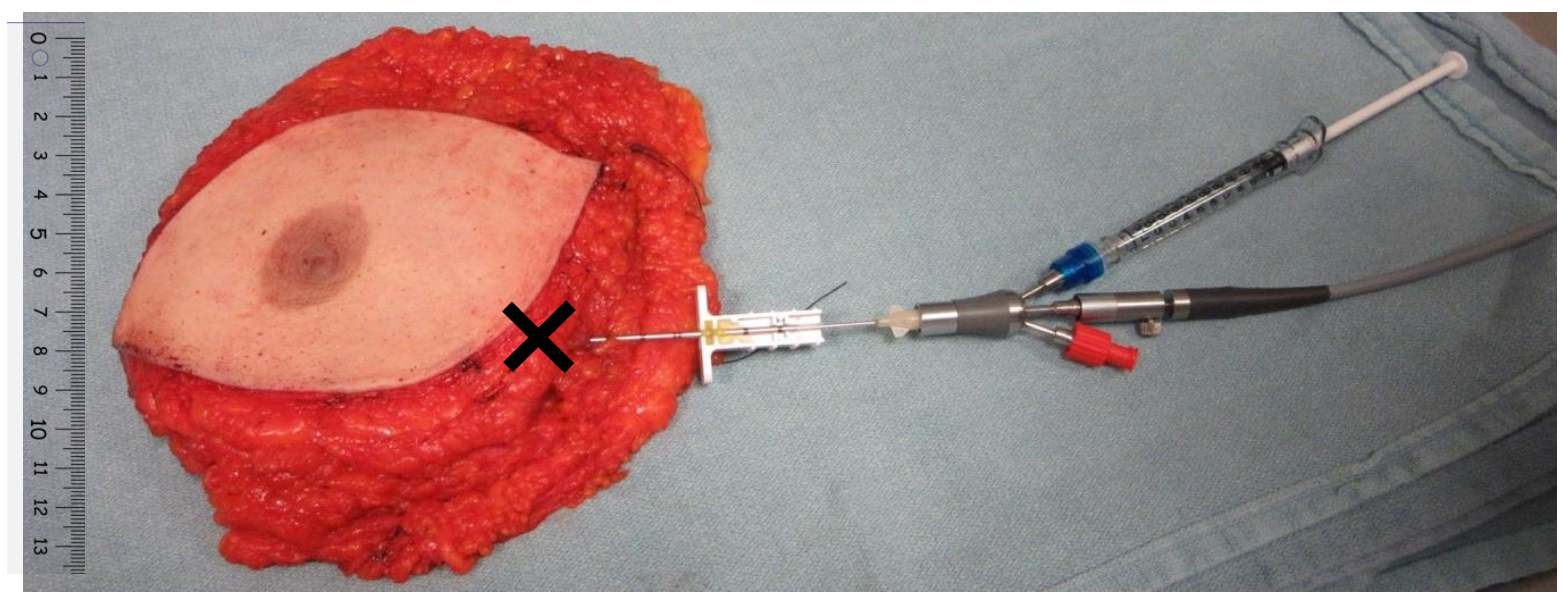

Figure 33: Data acquisition- Participant no. 1. Cross signifies the location of the tumor.

Observations: Tumor was easily situated as it was favorably palpable. Breast was highly firm and trocar insertion was a bit of a challenge. There was minimal bleeding during the procedure. Rinsing was not required as there was no inflow of blood into the trocar. Clear interstitial AF images was captured for all samplings. Patient had received an extensive neo-adjuvant treatment. Two dissected sentinel lymph nodes in the absence of patent blue dye were tested.

Challenge: In this experiment, biopsy samples were taken with biopsy forceps. However, the forceps weren't effective for acquiring proper amount of tissue. Considering the firmness of the tissue forceps jaws were too delicate. Secondly, trocar handle was too subtle and it broke during the insertion into the tumor.

Solution: For the remaining of the experiment a more durable type of trocar (BARD trocar with needle 12 gauge) and the more effective technique of core needle biopsy was employed. 


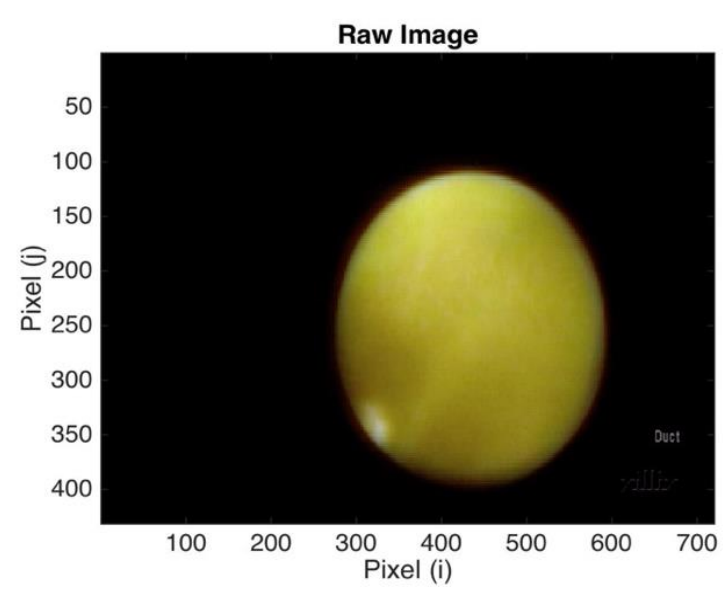

(a)

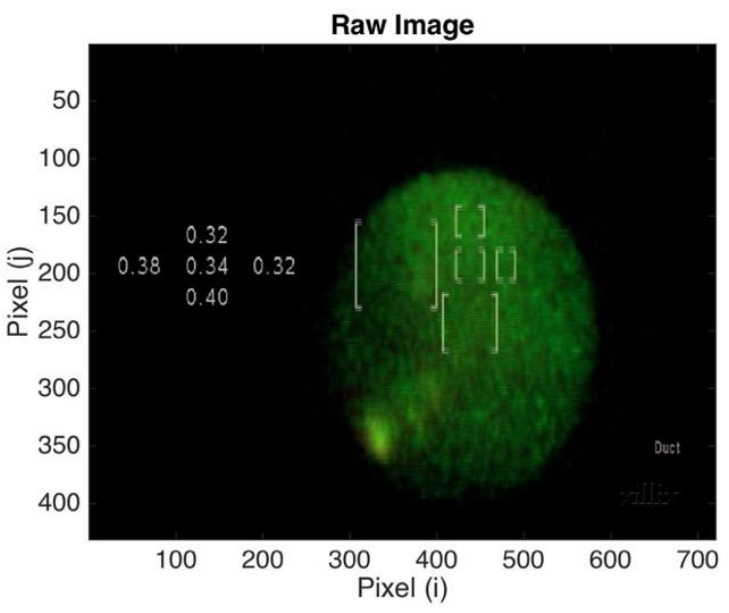

(b)

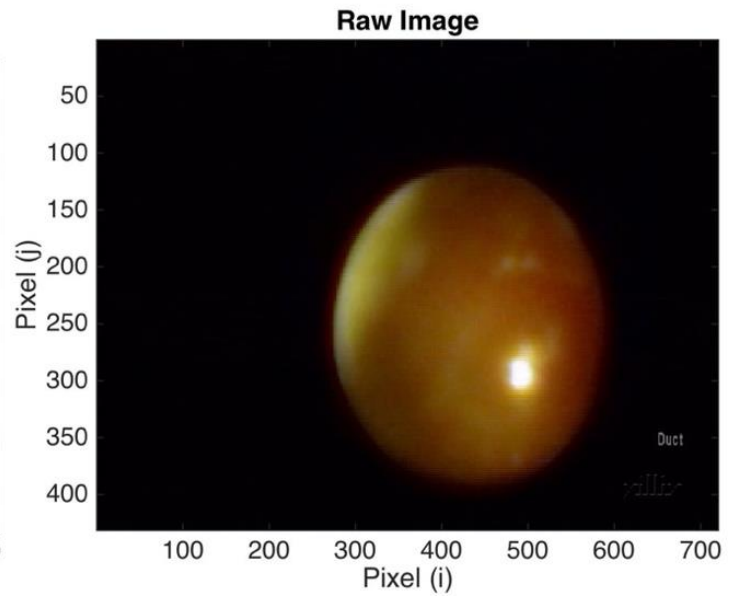

(c)

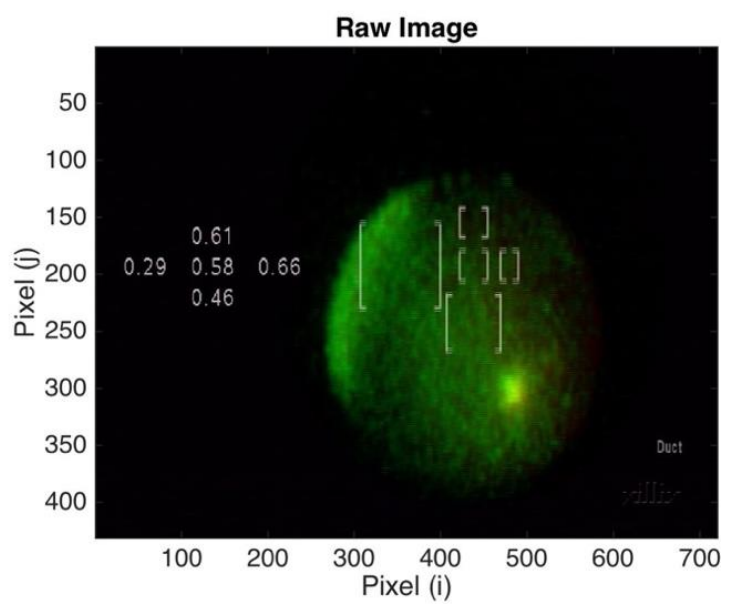

(d)

Figure 34: Case 1: Normal (a) white light and (b) autofluorescence compared to tumor (c) white light and (d) autofluorescence. The field of view has a diameter of approximately $2 \mathrm{~mm}$. The NCV locations and related values are shown in $b$ and $d$. 


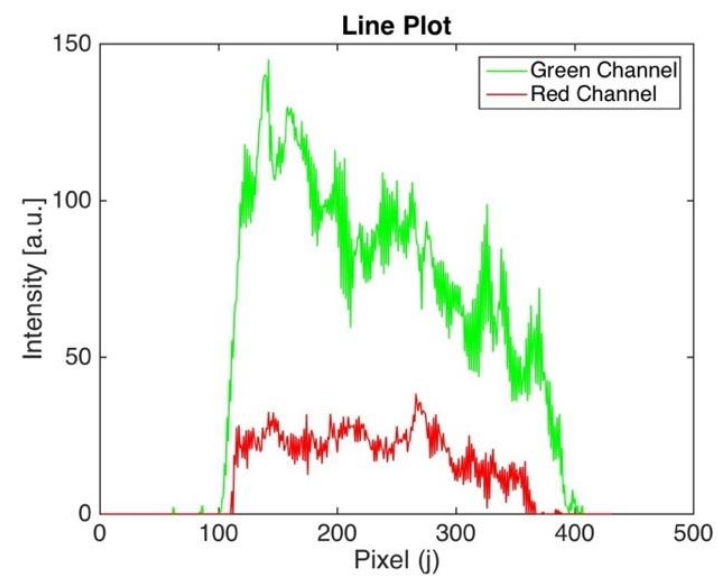

(a)

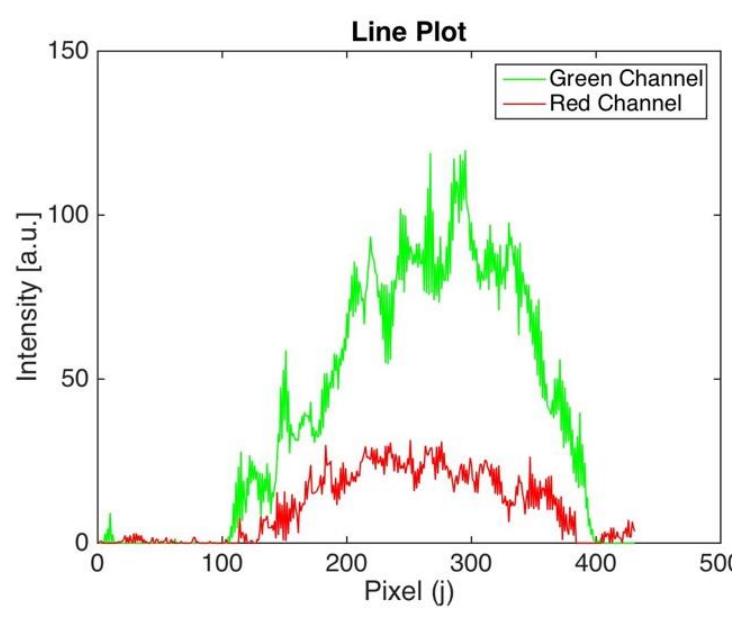

(b)

Figure 35: Case 1: Line plot of green, red and blue channels of autofluorescence images along the center of the image (i=450) for normal (a) and tumor (b)

Table 2: Pathology Assessment of Samples acquired for correlation purposes in the study for participant No.1

\begin{tabular}{|c|c|l|}
\hline & $\begin{array}{c}\text { Abnormal } \\
\text { Region }\end{array}$ & $\begin{array}{l}\text { Normal } \\
\text { region }\end{array}$ \\
\hline $\begin{array}{c}\text { Pathology Results of } \\
\text { Samples }\end{array}$ & Fibrosis tissue & $\begin{array}{l}\text { Normal } \\
\text { tissue }\end{array}$ \\
\hline
\end{tabular}

The patient was responsive to chemo and therefore tumor had shrunk extensively. As a result of the successful treatment, our data most likely was taken from normal and fibrosis tissue. Green colorization of AF images and low NCV values were compatible with the outputs of pathology analysis of the area. This indicate a complete response to neo-adjuvant chemotherapy and a successful detection of true negative for this imaging modality, confirmed by the pathologist. Reminding that the purpose of the study is to investigate the differences of $\mathrm{AF}$ images for normal and cancerous tissue and for this case, tumor was large and the initial pathology assessment of tumor indicated invasive carcinoma. As a result of the chemotherapy the cancerous tissue has dissipated which was first discovered by AF imaging and later on confirmed by the pathologist. Taking into account the possibility of chemotherapy to alter the pathological characteristic of the tumor and learning from this case, neo-adjuvant patients were excluded from the study after this patient and on. 


\subsection{Participant \#2}

\section{Pathology results of the entire tumor and lymph nodes:}

All lymph nodes negative

$2.2 \mathrm{~cm}$ Invasive $+4.9 \mathrm{~cm}$ extended DCIS

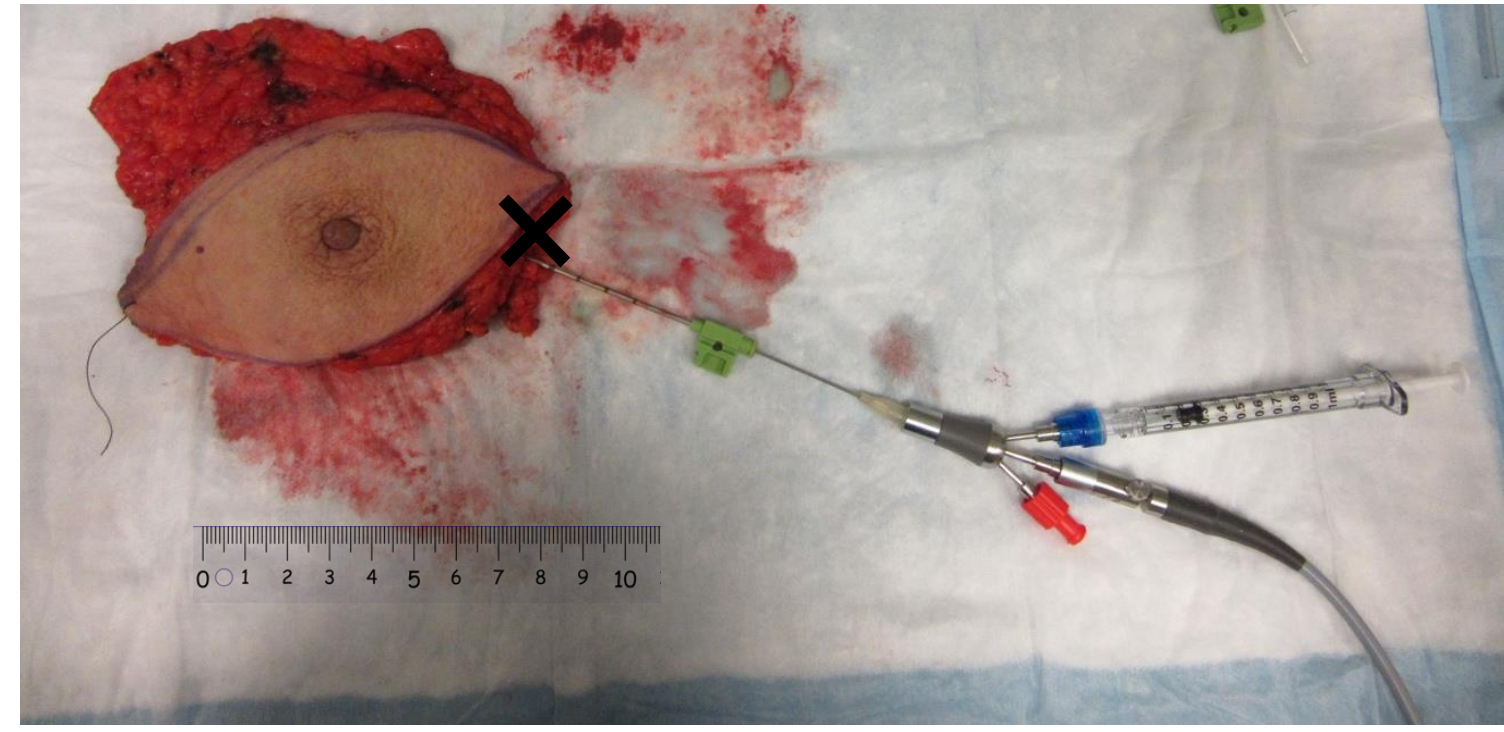

Figure 36: Data acquisition- Participant no. 2-cross signifies the location of the tumor

Observations: This experiment was initiated by testing the breast. Patient had a relatively small and highly vasculature breast. Tumor was $2 \mathrm{~cm}$ and palpable. The insertion was done at absence of ultrasound guidance. The tumor could be felt. Moving the needle was also moving the lump and therefore it confirmed that the tip of needle is correctly inside the tumor. The stylet was withdrawn and then the endoscope was inserted. AF videos were taken upon saline flushing of the channel otherwise blood was obscuring the image. Upon rinsing the channel, vivid green and reddish images were observed. Rinsing was repeated twice per examination. Subsequently, biopsy samples were taken using core needle biopsy.

Challenges: A relatively high hemorrhage was observed. As the result, the inflow of blood into the needle was substantial which was obscuring the image.

Solution: Upon ample rinsing a clear AF image was observed. 


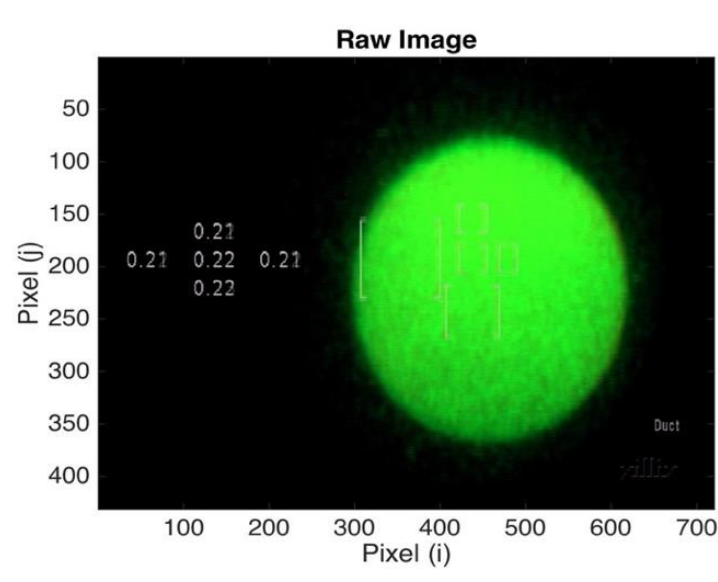

(a)

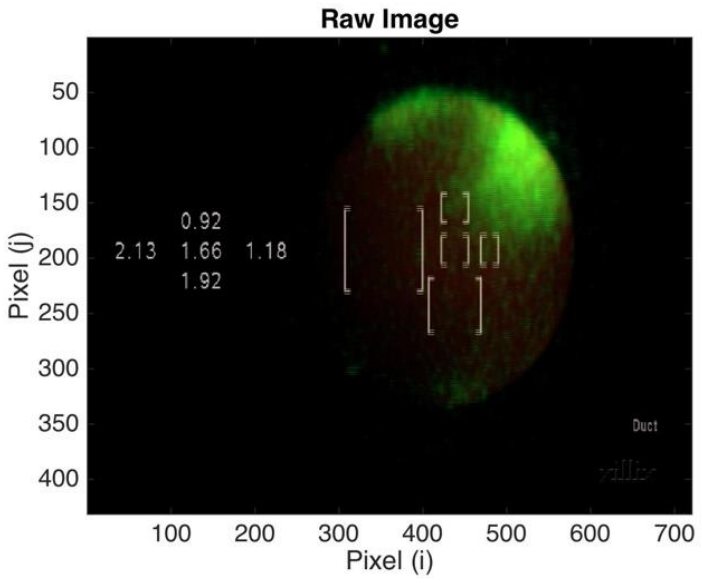

(b)

Figure 37: Case 2: Autofluorescence imaging of normal (a) versus malignant tumor (b). The field of view has a diameter of approximately $2 \mathrm{~mm}$. The NCV locations and related values are shown.

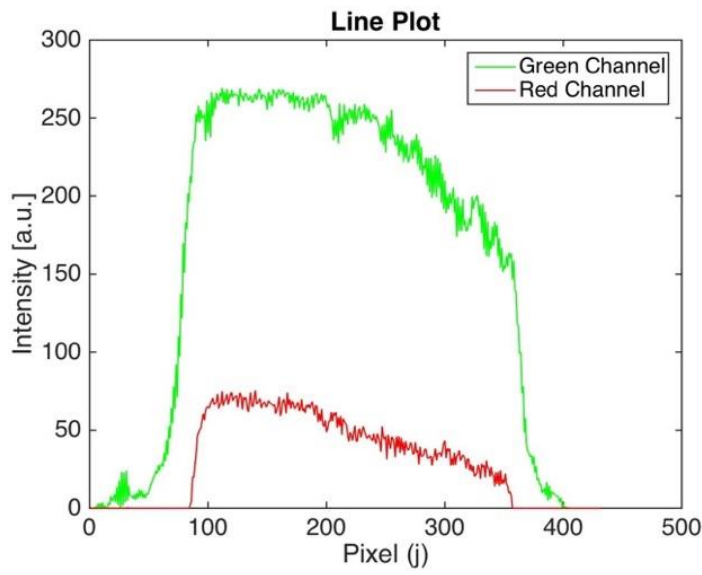

(a)

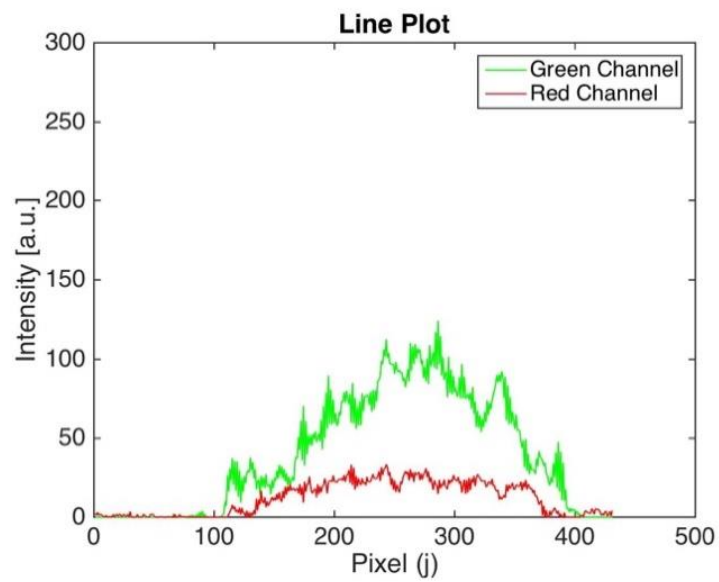

(b)

Figure 38: Case 2: Line plot of green, red and blue channels of autofluorescence images along the center of the image ( $i=450)$ for normal $(a)$ and tumor (b)

Table 3: Pathology Assessment of Samples acquired for correlation purposes in the study for participant No.2

\begin{tabular}{|c|c|l|}
\hline & $\begin{array}{c}\text { Abnormal } \\
\text { Region }\end{array}$ & $\begin{array}{l}\text { Normal } \\
\text { region }\end{array}$ \\
\hline $\begin{array}{c}\text { Pathology Results of } \\
\text { Samples }\end{array}$ & $\begin{array}{c}\text { In-situ Ductal } \\
\text { Carcinoma Tissue }\end{array}$ & $\begin{array}{l}\text { Normal } \\
\text { tissue }\end{array}$ \\
\hline
\end{tabular}

Pathological analysis of normal tissue showed no signs of malignancy which was compatible with the vivid green AF images. Intensity of the generated green is 
significantly higher than red and NCVs are below 0.3 , both indication of normal tissue. In contrary, for samples taken from abnormal tissue AF image has a range of red color, NCVs are higher than 0.8 and red and green channels have similar intensities. Pathology results showed Ductal Carcinoma In-Situ (DCIS). Correlation showed that AF images not only successfully discriminated normal and cancer but also detected In-situ carcinomas as a malignancy.

\subsection{Participant \#3}

\section{Pathology results of the entire tumor and lymph nodes:}

Invasive Ductal Carcinoma + Extensive DCIS

4 separate lumps: $1.7 \mathrm{~cm}-1.2 \mathrm{~cm}-1.4 \mathrm{~cm}-1.1 \mathrm{~cm}$

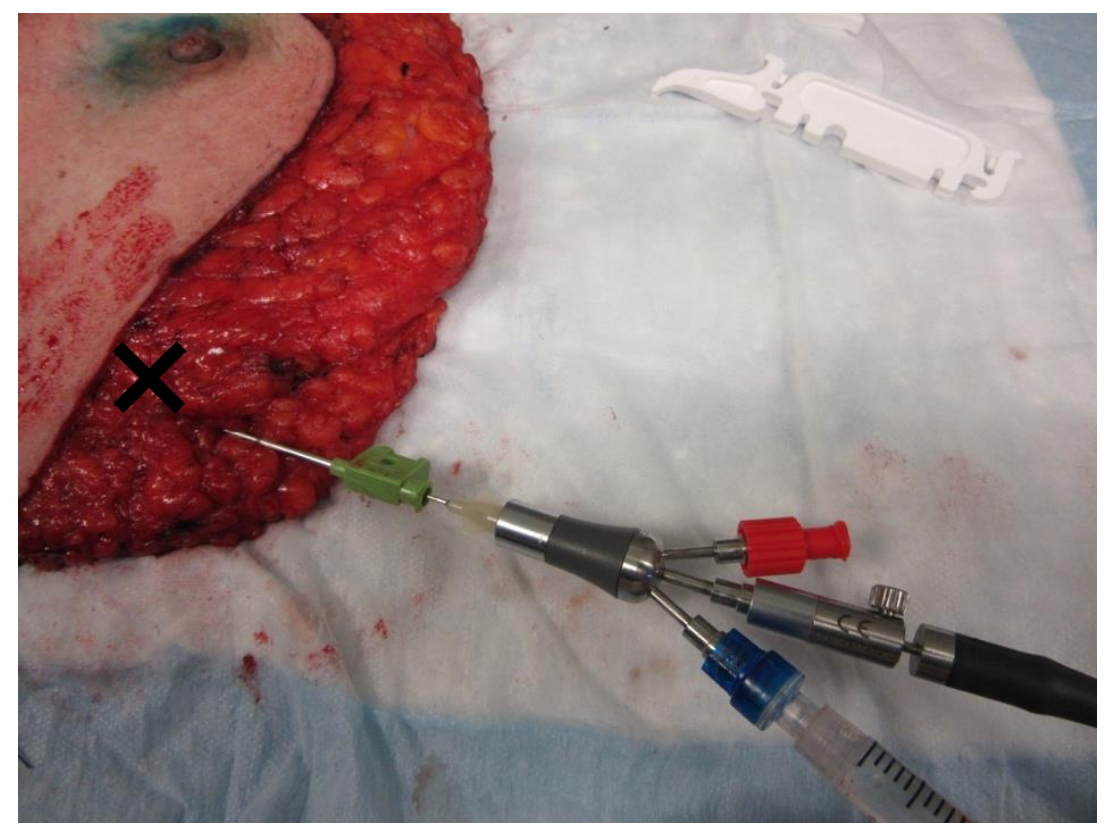

Figure 39: Data acquisition- Participant no. 3. Cross signifies the location of the tumor.

Observations: There were 3 sentinel lymph nodes. Red areas were observed for all, however pathology analysis showed no malignant tissue for all three. Patent blue dye has a relatively high absorption which will affect AF images. This was correctly anticipated and taken into account when recruiting the patients. However, patent blue dye was injected into the nipple around $4 \mathrm{~cm}$ away from the lump, it didn't enter the breast tumor and therefore had no effect on the AF images. There was no blood obscuring the image 
and consequently proper images in both WL and AF was observed. Upon rising a clear green image for normal and a reddish image for suspicious area was seen.

Challenges: breast was diffusively lumpy with multiple tumors and they were all fairly small. Locating the largest lump was a bit of a challenge. Samples were taken from the largest mass.

Solution: Locating the largest tumor and insertion was done by an experienced breast cancer surgeon.

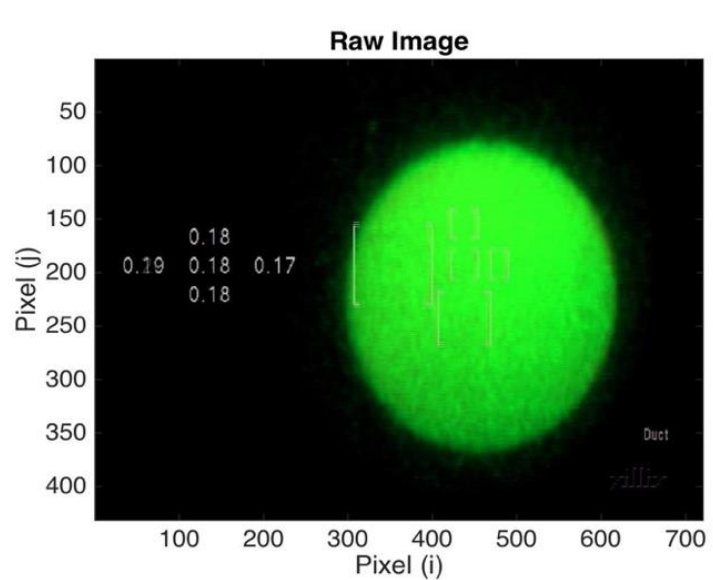

(a)

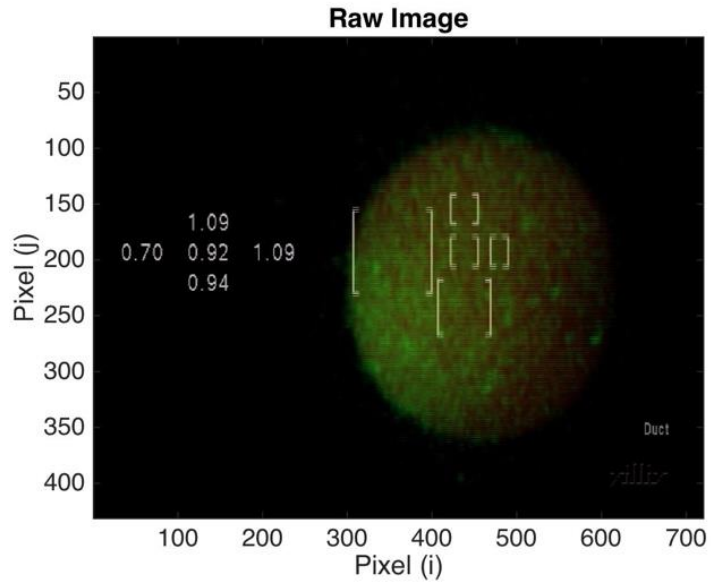

(b)

Figure 40: Case 3: Autofluorescence imaging of normal (a) versus malignant tumor (b). The field of view has a diameter of approximately $2 \mathrm{~mm}$. The NCV locations and related values are shown.

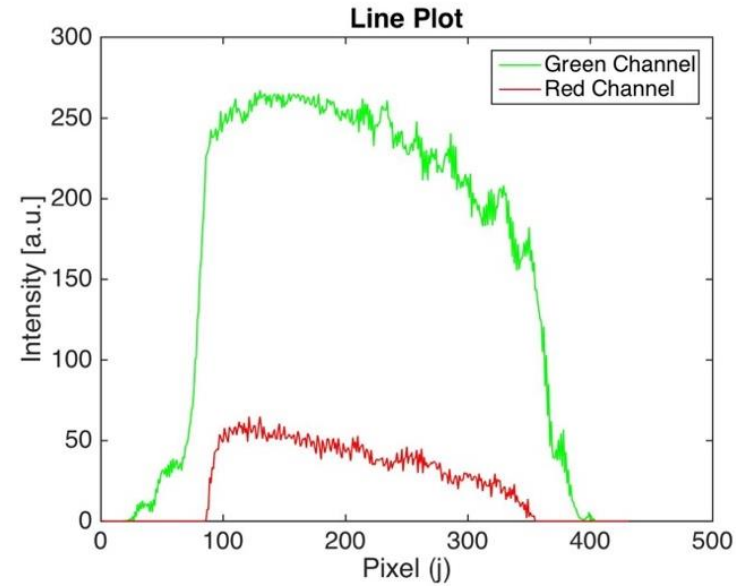

(a)

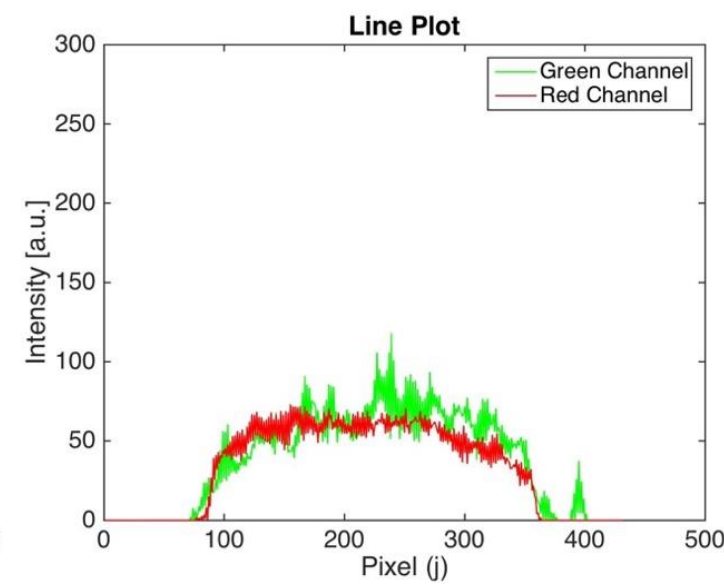

(b)

Figure 41: Case 3: Line plot of green, red and blue channels of autofluorescence images along the center of the image (i=450) for normal (a) and tumor (b)

Table 4: Pathology Assessment of Samples acquired for correlation purposes in the study for participant No.3

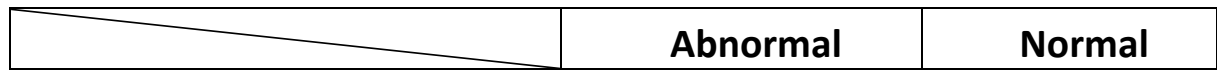




\begin{tabular}{|c|c|l|}
\hline & Region & region \\
\hline $\begin{array}{c}\text { Pathology Results of } \\
\text { Samples }\end{array}$ & $\begin{array}{c}\text { Invasive Ductal } \\
\text { Carcinoma tissue }\end{array}$ & $\begin{array}{l}\text { Normal } \\
\text { tissue }\end{array}$ \\
\hline
\end{tabular}

AF images correctly distinguished between normal and malignant tissues and NCVs of 0.18 and 0.92 was measured respectively. Results were confirmed by pathology results.

\subsection{Participant \#4}

\section{Pathology results of the entire tumor:}

Invasive Ductal Carcinoma- Tumor size $1.6 \mathrm{~cm}$

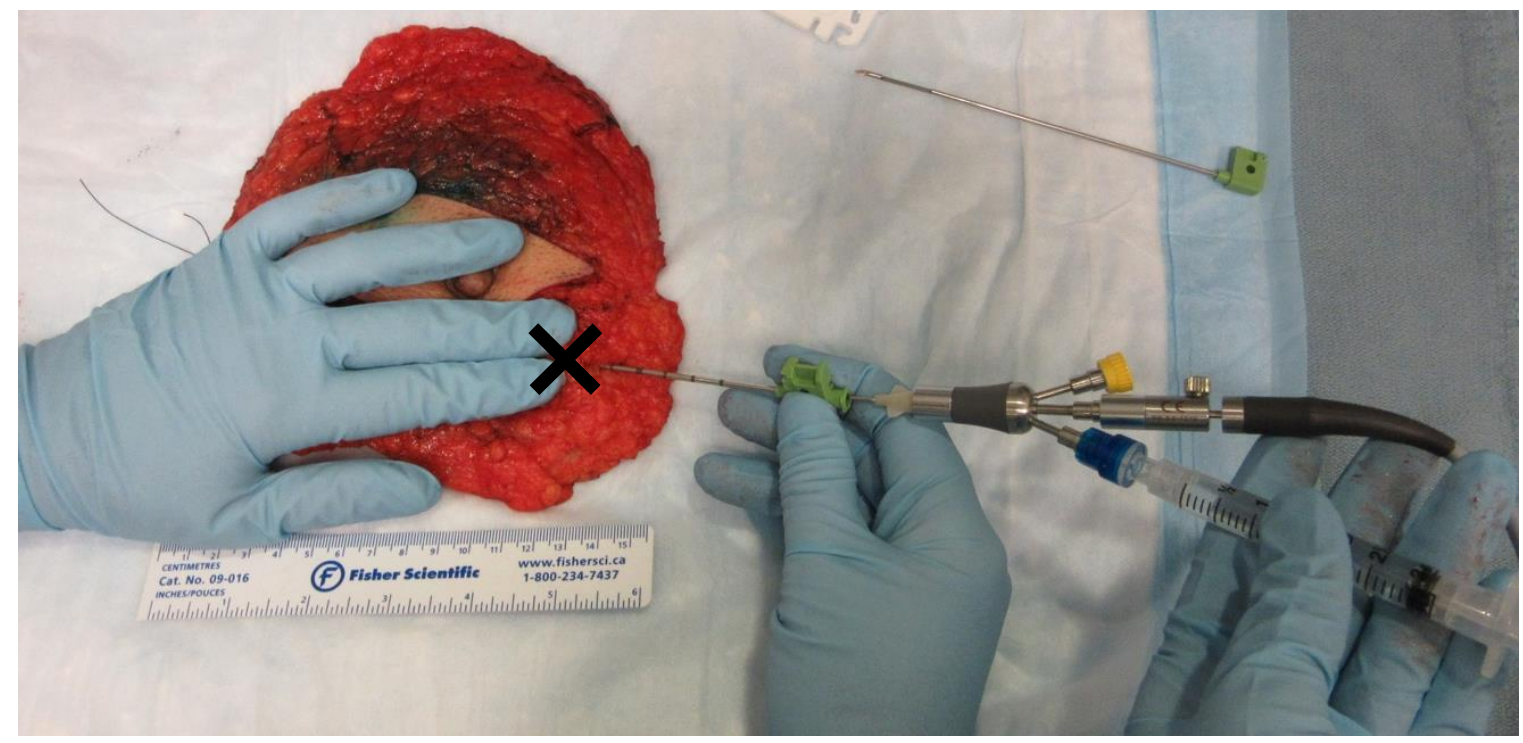

Figure 42: Data acquisition- Participant no. 4. Cross signifies the location of the tumor

Observations: Patient had received patent blue in here nipple. Clear interstitial images were collected. Holding the trocar inside was a big of a challenge and trocar kept sliding out which was mostly due to the small size of the tissue. Clear interstitial images were formed from both normal and tumor.

Challenges: Tumor was fairly small. Locating the tumor was not as straightforward.

Solution: Tumor was successfully located by an experienced breast cancer surgeon. 


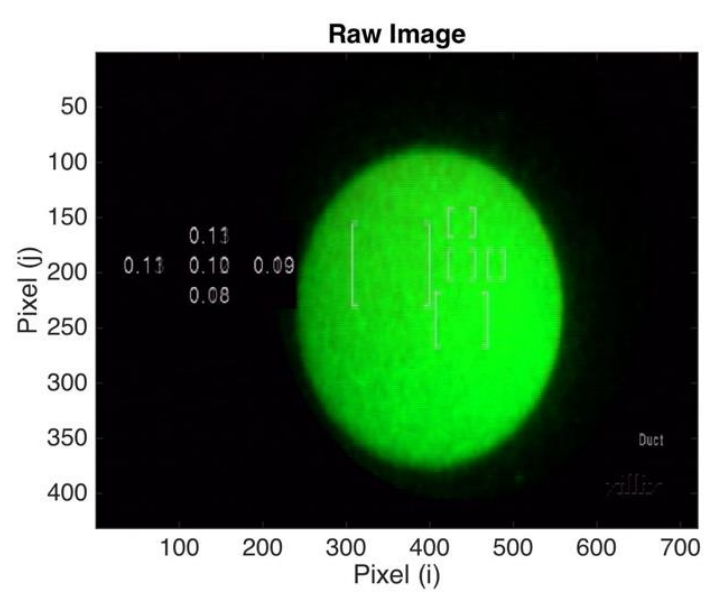

(a)

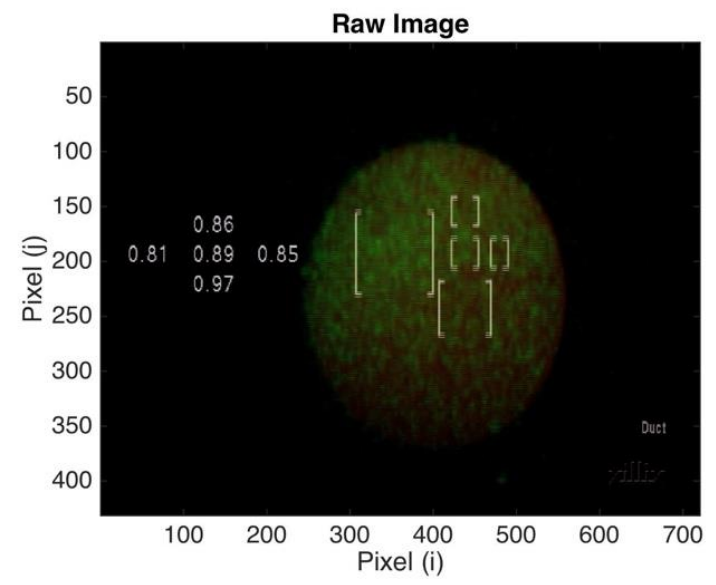

(b)

Figure 43: Case 4: Autofluorescence imaging of normal (a) versus malignant tumor (b). The field of view has a diameter of approximately $2 \mathrm{~mm}$. The NCV locations and related values are shown.

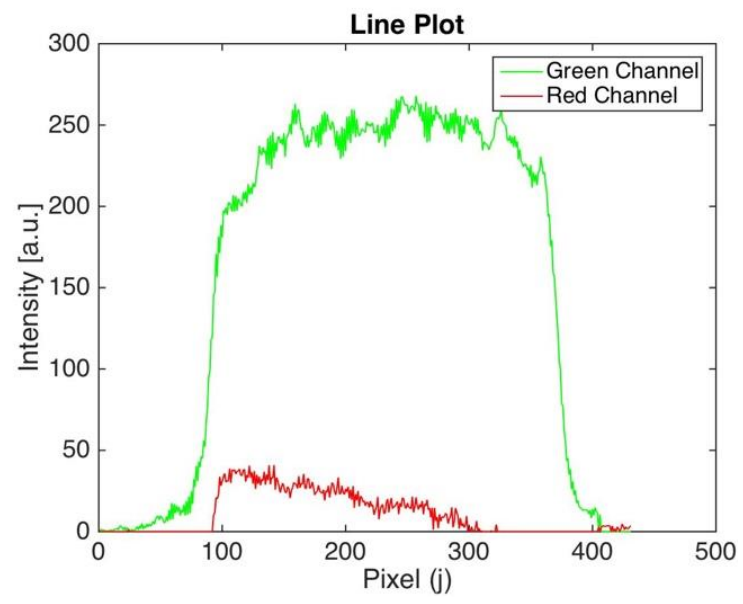

(a)

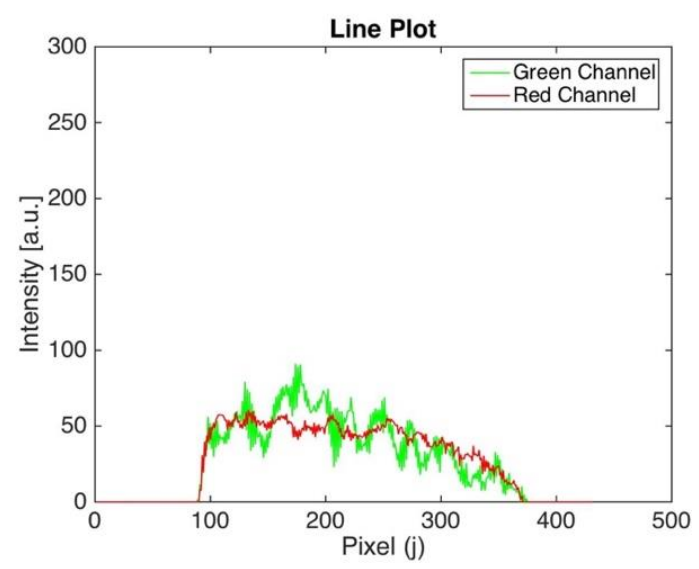

(b)

Figure 44: Case 4: Line plot of green, red and blue channels of autofluorescence images along the center of the image (i=450) for normal (a) and tumor (b)

Table 5: Pathology Assessment of Samples acquired for correlation purposes in the study for participant No.4

\begin{tabular}{|c|c|l|}
\hline & $\begin{array}{c}\text { Abnormal } \\
\text { Region }\end{array}$ & $\begin{array}{l}\text { Normal } \\
\text { region }\end{array}$ \\
\hline $\begin{array}{c}\text { Pathology Results of } \\
\text { Samples }\end{array}$ & $\begin{array}{c}\text { Invasive Ductal } \\
\text { Carcinoma tissue }\end{array}$ & $\begin{array}{l}\text { Normal } \\
\text { tissue }\end{array}$ \\
\hline
\end{tabular}


Case 4 was a success as AF images correctly showed biopsy proved healthy and malignant as green and reddish respectively. NCVs were also compatible and recorded 0.1 for normal and 0.89 .

\subsection{Participant \#5}

\section{Pathology results of the entire tumor:}

Invasive Lobular Carcinoma- Tumor size $1.7 \mathrm{~cm}$

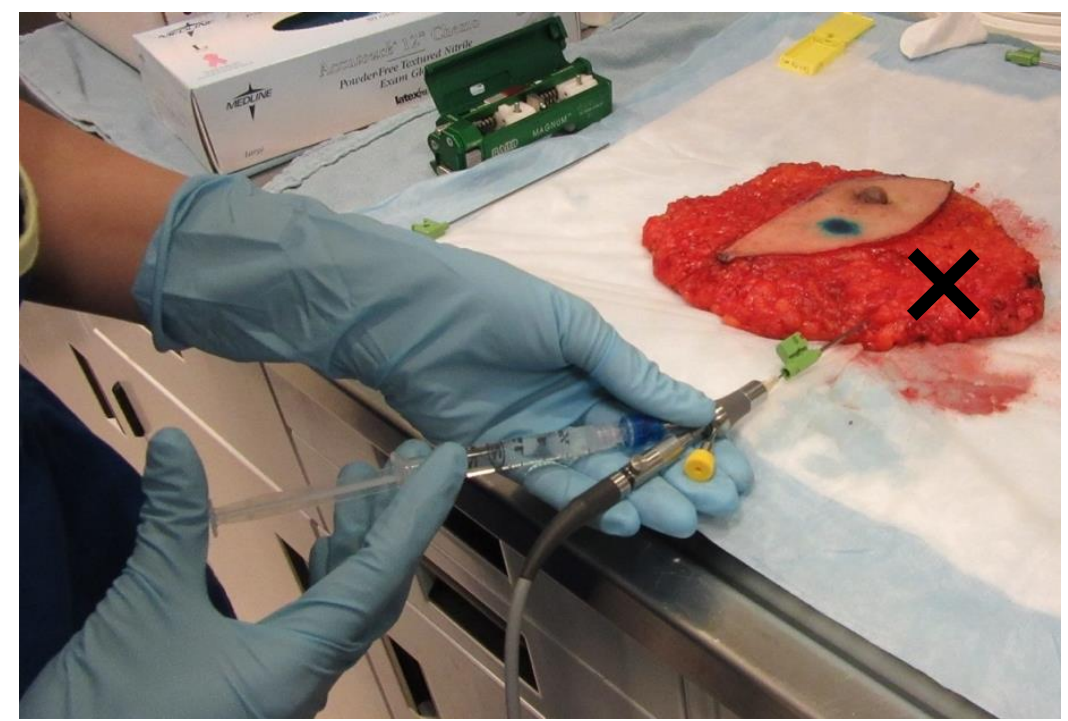

Figure 45: Data acquisition- Participant no. 5. Cross signifies the location of the tumor

\section{Observations:}

Patent blue was injected into the milk duct around $3 \mathrm{~cm}$ away from the tumor. Tumor were easily located and AF images from normal and tumor was acquired with no problem. 


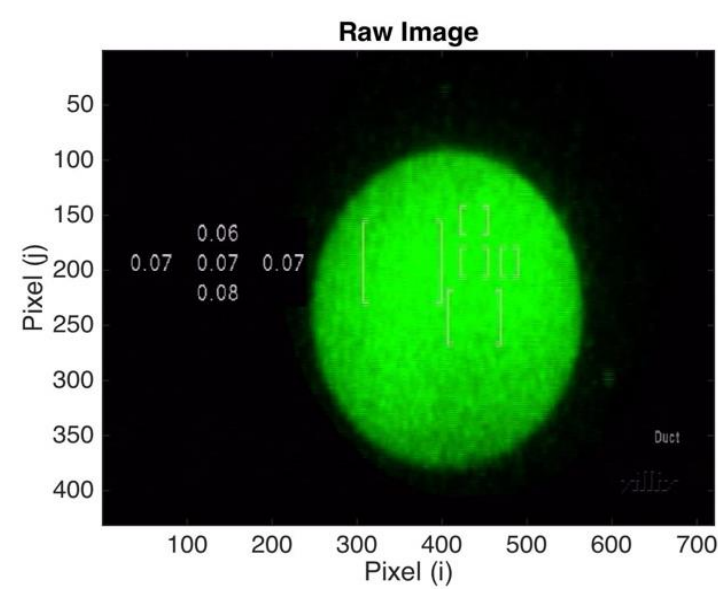

(a)

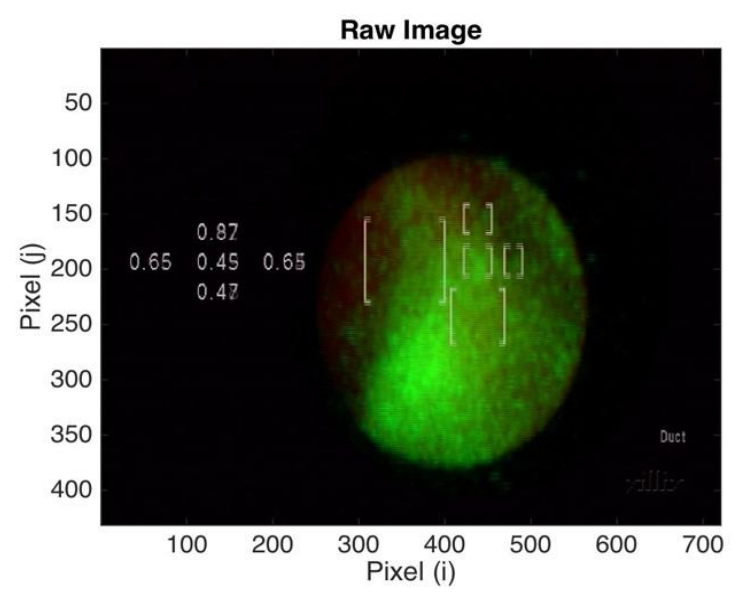

(b)

Figure 46: Case 5: Autofluorescence imaging of normal (a) versus malignant tumor (b). The field of view has a diameter of approximately $2 \mathrm{~mm}$. The NCV locations and related values are shown.

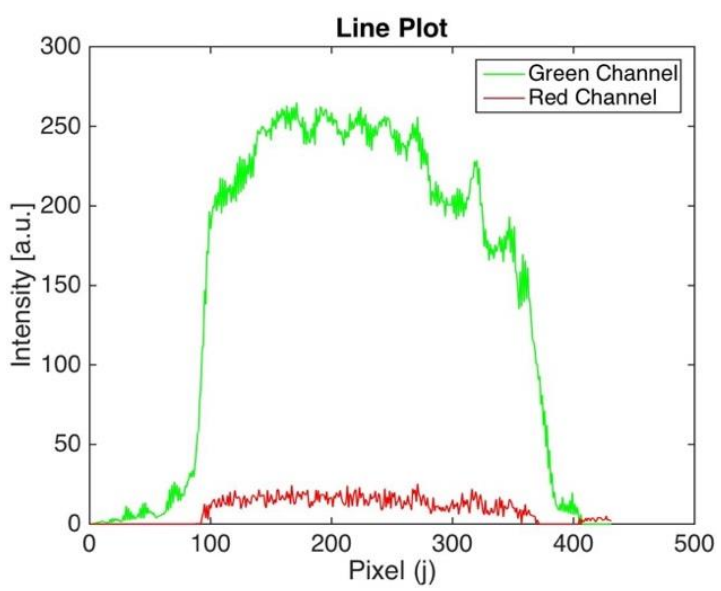

(a)

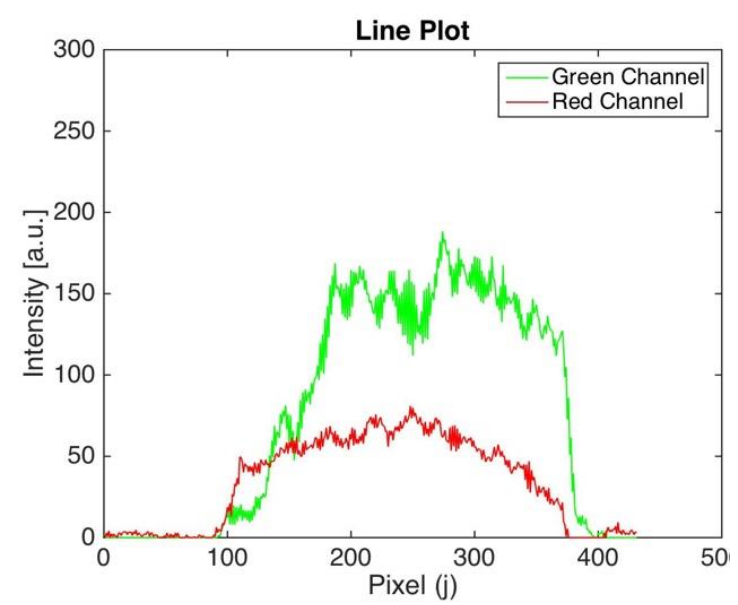

(b)

Figure 47: Case 5: Line plot of green, red and blue channels of autofluorescence images along the center of the image ( $i=450)$ for normal ( $a$ ) and tumor (b)

Table 6: Pathology Assessment of Samples acquired for correlation purposes in the study for participant No.5

\begin{tabular}{|c|c|l|}
\hline & $\begin{array}{c}\text { Abnormal } \\
\text { Region }\end{array}$ & $\begin{array}{l}\text { Normal } \\
\text { region }\end{array}$ \\
\hline $\begin{array}{c}\text { Pathology Results of } \\
\text { Samples }\end{array}$ & Suspicious tissue & $\begin{array}{l}\text { Normal } \\
\text { tissue }\end{array}$ \\
\hline
\end{tabular}

Case 5 was also a success for both normal and abnormal regions. Green AF images and a low NCV value (0.07) of the healthy area was confirmed by pathology results. For the 
abnormal area, pathology reported suspicious tissue which indicated that although tumor cells existed in the sample the quantity was not high enough to be categorized as malignant. AF images were more green than red and NCV was measured as 0.57 (below 0.8 ) and none were indication of malignancy. This was also in agreement with pathology outputs.

\subsection{Numerical Color Value:}

Although cancer and normal tissue appeared as two distinct colorizations in autofluorescence (AF) imaging, the diagnosis will be more accurate if an associated quantified value can be compared. The OncoLIFE image processor calculates a numerical color value (NCV) for 5 distinct areas on AF images presented by benchmarks (figure 46). NCV is the ratio of red to green channels and $16^{*} 12$ pixels on the centers are averaged over four frames and continuously displayed on the AF images. Larger NCV is an indication of low autofluorescence intensity which signify the malignancy. For each case an average of all 5 areas for normal and malignant was calculated. The spatial average is a proper representation of all NCVs correspond to 5 separate areas. The field of view has a diameter of $1 \mathrm{~mm}$. Considering that all the tumors were relatively large and the small field of view, the area understudy had a homogenous malignant characteristics and taking the average among the 5 areas is justified. The results are shown in figure 48 for normal and malignant tissues. Error bars are the standard deviations for each case.

Furthermore, to have an overall conclusion in terms of NCV values, they were averaged among all 5 cases. Values of 0.15 and 1.22 among all cases was obtained for normal and tumor respectively. For the average of healthy NCV the first case was excluded. First participant had undergone multiple rounds of chemotherapy that had altered the characteristics of the tissues substantially proved by pathology results. Additionally, while Pathological results of $1^{\text {st }}$ and $5^{\text {th }}$ cases did not indicate malignant, $1^{\text {st }}$ and $5^{\text {th }}$ cases were excluded from average NCV of malignant tissue. For all cancer tissues NCV values were higher than 0.8 . 


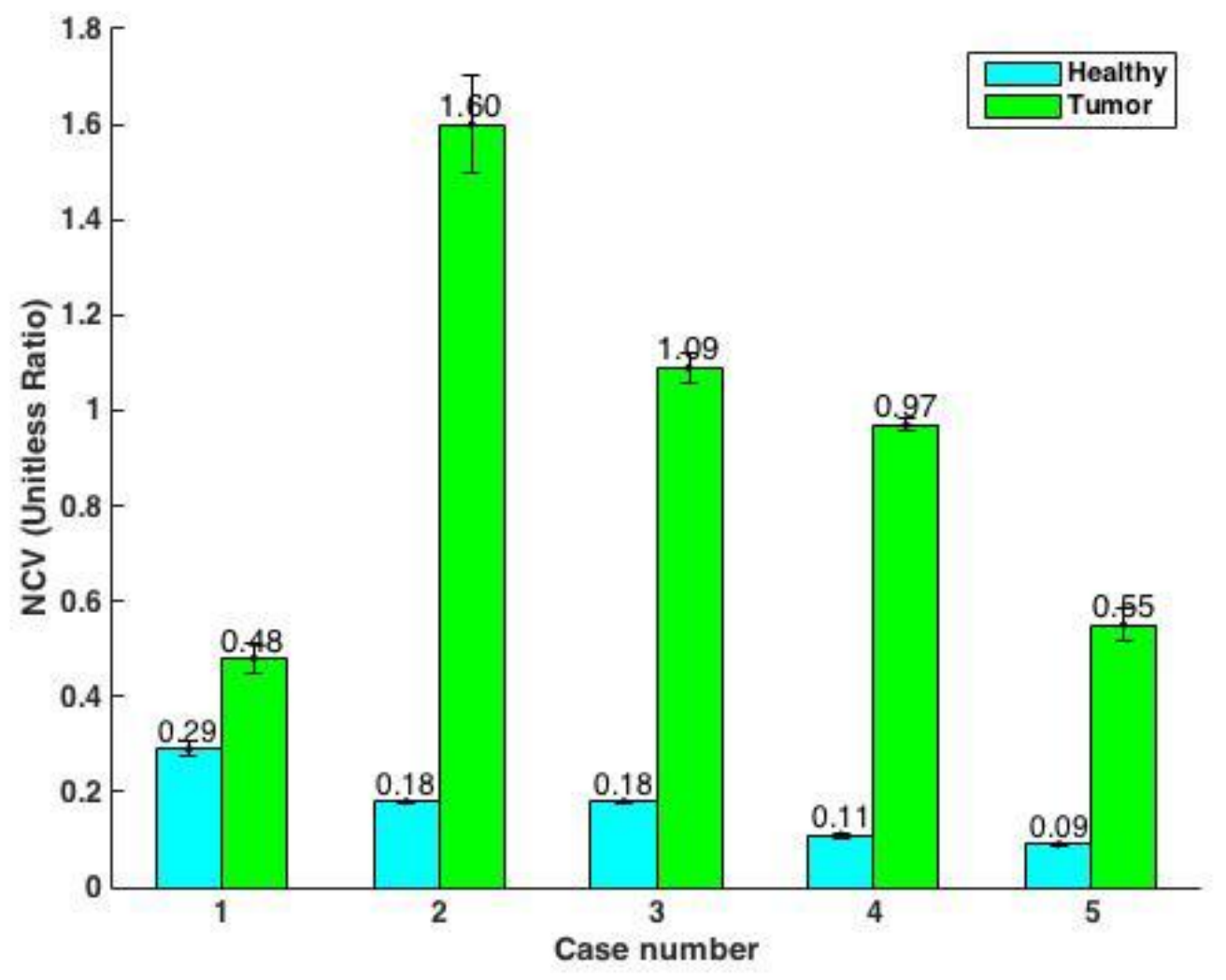

Figure 48: A comparison between the average NCVs of normal and malignant for all 5 cases and error bars

Table 7: Average, maximum and minimum of all 5 cases NCVs for normal versus cancer

\begin{tabular}{|c|c|c|c|}
\hline & Average NCV & Minimum & Maximum \\
\hline Healthy: & $0.14 \pm 0.07$ & 0.05 & 0.2 \\
\hline Tumor: & $1.22 \pm 0.33$ & 0.8 & 1.7 \\
\hline
\end{tabular}




\section{Chapter 4: Discussion and Conclusion}

In breast cancer diagnosis, there remain cases of uncertainty where existing diagnostic techniques in the absence of tools to directly visualize a suspicious mass inside a human body is problematic. Currently, ultrasound and mammogram are the prevailing clinical methods to clarify the diagnosis. However, tissue biopsy is the gold standard to confirm the malignancy. Based on the perspective interpretation of diagnostic images, radiologists classify the masses as benign or suspicious and judge if biopsy is required. The accuracy of this decision is highly dependent on the skills of the diagnostic radiologist. Biopsy is invasive, painful and costly. In instances where suspicious mass classifications and pathology results are not concordant, biopsy may need to be repeated.

Former work showed that autofluorescence imaging was reliable in distinguishing between cancer and normal in breast intraductally [6] [8]. However, ductal AF microendoscopy has limited prospective applications in management of breast cancer. Interstitial microendoscopy is more adaptable to the clinical needs and has a broader application. Interstitial AF images can directly visualize the abnormality and may be sufficient to be employed as a diagnostic signature when used coupled with biopsy. AF microendoscopy could potentially provide the diagnostic radiologist with more discriminatory information which may decrease the number of required biopsies. The ability of interstitial AF imaging coupled to a breast cancer biopsy tool can be confirmed as effective in an in-vivo clinical trial.

This study was a feasibility study emphasized on the capability of AF imaging on discriminating between normal and malignant interstitially on ex-vivo female breast tissue. This study is a preliminary phase of the actual in-vivo clinical trial. The developed apparatus along with an improved microendoscope compared to the previous ductal study was effective in acquiring clear interstitial images with proper resolution. The interstitial microendocope was semi-rigid has a better resolution and smaller diameter $(0.55 \mathrm{~mm})$. The newer generation of microendoscope has a suitable size to be fit inside a biopsy trocar and therefore can be coupled to biopsy. This feasibility study proved the ability of AF imaging to clearly visualize and correctly discriminate between normal and malignant breast tissues in all 5 ex-vivo cases. 
The biggest obstacle in interstitial microendoscopy of both ex-vivo and in-vivo tissues is the inflow of blood into the working channel that will obscure the image (blood absorbs visible light significantly). An efficient solution was to flush out the blood by means of saline using the setup shown in figure 26. AF images of both normal and tumor were taken accordingly. The developed interstitial system is feasible, practical and used existing equipment in the clinic.

Lastly, core needle biopsy samples of the area under study was acquired. Biopsies were used as the gold standard and AF images of both normal and cancer was correlated to the pathology results. Accurately locating the suspicious mass was possible due to palpability of tumors. The preliminary study showed that the routinely used biopsy trocar in radiology can also be employed in creating the interstitial channel. In this study a system was developed using the existing instrument in radiology that can easily be incorporated into a clinical setting and we are planning on that.

Excised tissues have intrinsically a large variation of tissue structure and other characteristics which is a challenge to overcome in an ex-vivo study. Standard operational procedure was proven effective for all 5 cases with various tissue traits. Firstly, trocar was sufficiently durable for insertion into firm breasts. Secondly, Interstitial AF was able to capture clear images for a range of highly vasculature tissue where inflow of blood into interstitial channel was dramatic in cases with significant hemorrhage. Biopsy samples were successfully taken for all cases using core needle biopsy by attaching a biopsy gun to the trocar. In AF imaging for all study cases, the cancer appeared as a domination of red color and the normal as a vivid green color. Additionally, data was quantified by the numerical color value (NCV). Study results showed that NCV values of higher than 0.8 are highly suggestive of malignancy. For all cases NCV of 0.3 and below was measured for healthy tissue.

Every single AF image was concordant with histopathology results. Outcomes showed a successful true negative when patient had received neo-adjuvant chemotherapy that dissipated the tumor substantially and only fibrosis tissue were detected. For this case AF successfully detect the fibrosis tissue as non-malignant and the measured NCV was below 0.8. Two correct invasive ductal carcinomas were detected with NCV values of 
1.09 and 0.97. AF was sensitive enough to recognize in-situ carcinomas as malignant with NCV of 1.6.

Lymph nodes of 3 patients were tested. For patients that had received patent blue into their nipple, dye was abundant in the node. AF imaging of those nodes showed malignancy in all cases while the pathology results came back negative. Hence it can be concluded that AF is not diagnostically accurate in presence of patent blue dye.

\subsection{Future Work}

While the feasibility study was a success the next step is to investigate AF imaging diagnostic accuracy in-vivo. For future studies, the same procedure can be studied in-vivo since the standard operational procedure was designed using the existing equipment in radiology, also saline rinsing is permitted in-vivo. The microendoscope will be pass down the biopsy trocar prior to taking samples in radiology. The inflow of blood into the interstitial channel was not an issue in the ex-vivo study. However, due to the blood pressure it might be a greater obstacle in-vivo. Although for all cases tumor was successfully located due to palpability the accuracy of insertions can be further improved by ultrasound guidance. Additionally, there might be a threshold NCV value beyond which is highly suggestive of malignancy. To make a robust conclusion in this regard, a bigger sample size is required.

The quality of images using the microendoscope sufficed the requirement of this study while in all AF images a distinction between normal and malignant was possible. However, an endoscope with a better resolution (more than 6000 pixel) can improve the quality and resolution of images and the endoscope upgrade be required in the in-vivo study.

Autofluorescence imaging can only be established as a diagnostic coupling tool to breast cancer biopsy if proves effective in an in-vivo clinical study with a proper sample size. The clinical study should be designed so that AF images can be correlated to biopsy results and compared to the ultrasound interpretation of areas understudy. Unlike this current study where only biopsy proven healthy and malignant tissues were study, in that 
clinical study a large variation of suspicious tissues with different degrees of abnormality should be investigated. 


\section{Bibliography}

[1] Canadian cancer society, "Canadian cancer statistic," 2015.

[2] American Cancer Society, "What are the key statistics about breast cancer," Nov 2013. [Online]. Available: http://bit.ly/bknuel.

[3] M. Eberl, C. Fox, S. Edge, C. Carter and M. Mahoney, "BI-RADS Classification for Management of Abnormal Mammograms," JABFM, p. 10.3122/jabfm.19.2.161, 2006.

[4] S. Zanati, N. Marcon and M. Cirocco, "Onco-LIFE Fluorescence imaging during colonoscopy assists in the differentiation of adenomatous and hyperplastic polyps and improves the detection rate of dysplastic lesions of the colon," Gastroenterol, pp. 128, A27-28, 2005.

[5] A. Douplik, S. Zanati, G. Saiko, C. Streutker, M. Loshchenov, D. Adler, S. Cho, D. Chen, C. Cirocco, N. Marcon, J. Fengler and B. Wilson, "Diffuse reflectance spectroscopy in Barrett's esophagus:developing a large field-of-view screening methoddiscriminating dysplasia from metaplasia," J Biophotonics, 2012.

[6] A. Douplik, A. Leong, A. Easson, S. Done, G. Netchev and B. Wilson, "Feasibility study of autofluorescence mammary ductoscopy," J. Biomed. Opt., p. 10.1117/1.3210773, 2009.

[7] A. Douplik, W. Leong, A. Easson, A. Done, G. Netchev and B. Wilson, "Preliminary experience of autofluorescence mammary ductoscopy," Biomed Optics Med Imaging SPIE, Oct 2011.

[8] A. Douplik, W. Leong, A. Easson and e. al, "Microendoscopic spectral imaging as a tool for small ductal diagnostics: preliminary experience," Journal of Innovative Optical Health Sciences (JIOHS), pp. 05: 1250021 1-7, 2012.

[9] A. Kalitzeos, A. Zam, F. Stelzle, E. Hahn, M. Raithel and A. Douplik, "Enhancement of Cancerous/Normal Tissue Contrast viaCombined White Light and Fluorescence Image Processing initial," Biomedical Optics, p. 10.1117/12.831771, 2009.

[10] D. Berna, V. Garcia-Medina and C. Kuni, "Ductoscopy: a new technique for ductal exploration," Eur J Radiol, pp. 12:127-9, 1991.

[11] W. Dooley, D. Francescatti, L. Clark and e. a. , "Office-based breast duc- toscopy for diagnosis," Am J Surg , pp. 188:415-8, 2004.

[12] J. Simpson, E. Connolly, W. Leong, J. Escallon, D. McCready, M. Reedijk and A. Easson, "Mammary ductoscopy in the evaluation and treatment of pathologic nipple discharge: a Canadian experience," Can J Surg, 2009.

[13] E. N. Marieb, Essentials of Human Anatomy \& Physiology, 8th, Ed., San Fransisco, CA: Pearson Benjamin Cummings, 2006.

[14] American Cancer Society, "Breast Cancer," American Cancer Society, Atlanta, 2009.

[15] W. Roger and P. Williams, Angiology (Chapter 6)". Gray's anatomy. illustrated by 
Richard E. M. Moore, London: Longman, 1973, p. pp. 588-785..

[16] A. Stuckey, "Breast cancer: epidemiology and risk factors," Clin Obstet Gynecol., pp. 54(1):96-102., 2011.

[17] Surveillance Epidemiology and End Results, "SEER Stat Fact Sheets: Breast Cancer," Nov 2013. [Online]. Available: http://1.usa.gov/10AWIY .

[18] R. Siegel, D. Naishadham and A. Jemal, "Cancer statistics, 2013," CA Cancer J Clin., pp. 63(1):11-30., Jan 2013.

[19] S. Sanati and D. Allred, The Progression of Pre-invasive to Invasive Cancer. In: Fitzgerald R. ed. Pre-Invasive Disease: Pathogenesis and Clinical Management., New York: Springer Science+Business Media, LLC, 2011.

[20] NCCN Guidelines, "Breast Cancer Screening and Diagnosis, version 2.2013," Nov 2013. [Online]. Available: http://bit.ly/1i5VknM.

[21] Breast Cancer Screening and Diagnosis Guidelines, "National Comprehensive Cancer Network," Nov 2013. [Online]. Available: http://bit.ly/IdmpWf.

[22] J. Bougheya, R. Gonzalezb, E. Bonnerc and H. Kuererb, "Current Treatment and Clinical Trial Developments for Ductal Carcinoma In Situ of the Breast," Journal of translational oncology, pp. 10.1634/theoncologist.12-11-1276, 2007.

[23] J. Lakowicz, Principle of Fluorescence Spectroscopy, 3rd ed., Baltimore, MC: Springer, 2006.

[24] M. Sauer, J. Hofkens and J. Enderlein, Handbook of Fluorescence Spectroscopy and Imaging, Weinheim: WILEY-VCH Verlag GmbH \& Co, 2011.

[25] D. J. Griffiths, Introduction to Quantum Mechanics, Prentice Hall. ISBN 0-13111892-7., 2004.

[26] P. N. Prasad, Introduction to biophotonics, Hoboken, NJ: John Wiley \& Sons, Inc., 2003.

[27] J. Mannekutla, B. Mulimani and S. Inamdar, Solvent effect on absorption and fluorescence spectra of coumarin laser dyes: Evaluation of ground and excited state dipole moments, Spectrochimica Acta Part A: Molecular and Biomolecular Spectroscopy, 2007, p. 10.1016/j.saa.2007.04.016.

[28] D. B. Jakubowski, A. E. Cerussi, F. Bevilacquia, N. Shah, D. Hsiang, J. Butler and B. J. Tromberg, "Monitoring neoadjuvant chemotherapy in breast cancer using quantitative diffuse optical spectroscopy: a case study," J. Biomed. Opt. , p. 9230 8, 2004.

[29] M. Homocianu, A. Airinei and D. Dorohoi, "Solvent Effects on the Electronic Absorption and Fluorescence Spectra," Journal of Advanced Research in Physics, pp. 2(1), 011105, 2011.

[30] M. Kasha, "Characterization of electronic transitions in complex molecules," Disc Faraday Soc, p. 142:463-562, 1950.

[31] A. S. Coolidge, H. M. James and R. D. Present, "A study of the Franck-Condon Principle," Journal of Chemical Physics, p. 10.1063/1.1749818, 1936. 
[32] J. ". Franck, "Elementary processes of photochemical reactions," Transactions of the Faraday Society, p. 21: 536-542, 1926.

[33] A. Coolidge, H. M. James and R. D. Present, "A study of the Franck-Condon Principle," A study of the Franck-Condon Principle, p. 4: 193-211, 1936.

[34] F. Bernath, Spectra of Atoms and Molecules(Topics in Physical Chemistry), Oxford: Oxford: Oxford University Press, 1995.

[35] E. Condon, "Nuclear motions associated with electron transitions in diatomic molecules," Physical Review, p. 10.1103/PhysRev.32.858, 1928.

[36] P. Atkins and R. S. Friedman, Molecular Quantum Mechanics., Oxford: Oxford University Press, 1999.

[37] G. G. Hammes, Spectroscopy for the Biological Sciences, Whiley-VCH , 2005.

[38] M. Monici, "Cell and tissue autofluorescence research and diagnostic applications," Biotechnol Annu. Rev, pp. 10.1016/S1387-2656(05)11007-2, 2005.

[39] N. Ramanujam, "Encyclopedia of Analytical Chemistry R.A. Meyers (Ed.)," in Fluorescence Spectroscopy In Vivo, Chichester, John Wiley \& Sons Ltd, 2000, p. pp. 20-56.

[40] A. Katz and A. R, "Non-invasive Fluorescence-based instrumentation for cancer and precancer detection and screening," In-vitro Dianostic Instrumentation, p. 10.1117/12.382035, 2000.

[41] M. Fang, J. Yuan, C. Peng and Y. Li, "Collagen as a double-edged sword in tumor progressio," Tumour Biol. , p. 35(4): 2871-2882, 2014.

[42] L. Marcu, P. French and D. Elson, Fluorescence Lifetime Spectroscopy and Imaging: Principles and Applications in biomedical diagnosis, New York: CRC press, 2015.

[43] I. Flamme, T. Frölich and W. Risau, "Molecular mechanisms of vasculogenesis and embryonic angiogenesis," Journal of cellular physiology, p. Journal of cellular physiology, 1997.

[44] N. Nishida, H. Yano, T. Nishida, T. Kamura and M. Kojiro, "Angiogenesis in Cancer," Vasc Health Risk Manag, p. 2(3): 213-219, 2006.

[45] S. Cohen, Biophotonic in Pathology, New Jersey : IOS press, 2013.

[46] S. L. Jacques, "Optical properties of biological tissues: a review," Phys. Med. Biol. 58 (2013) R37-R61, p. R57, 2013.

[47] P. Werahera, E. Jasion, Y. Liu, J. Daily, P. Arangua, C. Jones, S. Nash, M. Morrell and E. Crawford, "Human feasibility study of fluorescence spectroscopy guided optical biopsy needle for prostate cancer diagnosis," Eng Med Biol Soc. , no. doi: 10.1109/EMBC.2015.7320091, pp. 7358-61, 2015.

[48] B. Li and S. Xie, "Autofluorescence excitation-emission matrices for diagnosis of colonic cancer," World Journal of Gastroenterology, pp. 11(25):3931-3934, 2005.

[49] J. Lennard-Jones, J. Ritchie, B. Morson and C. Williams, "Cancer surveillance in ulcerative colitis: Experience over 15 years," Lancet, pp. 149-152, 1983.

[50] E. Rosenstock, R. Farmer, R. Petras, M. Sivak, G. Rankin and B. Sullivan, 
"Surveillance for colonic carcinoma in ulcerative colitis," Gust. End, pp. 1342-1346,, 1985.

[51] G. Bottiroli, A. Croce, D. Locatelli, R. Marchesini, E. Pignoli, S. Tomatis, C. Cuzzoni, S. Di Palma, M. Dalfante and P. Spinelli, "Natural fluorescence of normal and neoplastic human colon: a comprehensive "ex vivo" study," Lasers Surg Med. , pp. 16(1):48-60, 1995.

[52] M. Karita, D. Cantero and K. Okita, "Endoscopic diagnosis and resection treatment for flat adenoma with severe dysplasia," Amer. J. Gastroenterol, pp. 1421-1423, 1993.

[53] H. Aihara, H. Tajiri and T. Suzuki, "Application of Autofluorescence Endoscopy for Colorectal Cancer Screening: Rationale and an Update," Gastroenterology Research and Practice, p. dx.doi.org/10.1155/2012/971383, 2011.

[54] H. Tajir, "Autofluorescence endoscopy for the gastrointestinal tract," Phys Biol Sci. , p. 83(8): 248-255., Dec 2007.

[55] M. Kara, F. Peters, F. Ten Kate, S. Van Deventer, P. Fockens and J. Bergman, "Endoscopic video autofluorescence imaging may improve the detection of early neoplasia in patients with Barrett's esophagus.," Gastrointest Endosc, pp. 61(6):67985, 2005.

[56] S. Zanati, N. Marcon, M. Cirocco, N. Basset, C. Streutker, G. Kandel, P. Kortan, S. Rychel, A. Douplik and B. Wilson, "Onco-LIFE Fluorescence Imaging during Colonoscopy Assists in the Differentiation of Adenomatous and Hyperplastic Polyps and Improves the Detection Rate of Dysplastic Lesions in the Colon," Gastroenterology, pp. 128, A27-8, 2006.

[57] M. Scott, C. Hopper, A. Sahota, R. M. B. Springett, S. Bown and A. MacRobert, "Fluorescence Photodiagnostics and Photobleaching Studies of Cancerous Lesions using Ratio Imaging and Spectroscopic Techniques," Lasers Med Sci, p. 15:63-72, 2000.

[58] G. Bottiroli, A. Croce, D. Locatelli, R. Marchesini, E. Pignoli, S. Tomatis, C. Cuzzoni, S. Di Palma, M. Dalfante and P. Spinelli, "Natural Fluorescence of Normal and Neoplastic Human Colon: A Comprehensive "Ex Vivo" Study," Lasers in Surgery and Medicine, pp. 1648-60, 1995.

[59] R. Ishihara, T. Inoue, N. Hanaoka, Y. Takeuchi, Y. Tsujii, H. Kanzaki, T. Oota, M. Hanafusa, S. Yamamoto, K. Nagai and F. Matsui, "Autofluorescence imaging endoscopy for screening ofesophageal squamous mucosal high-grade neoplasia:A phase II study," Journal of Gastroenterology and Hepatology, pp. 10.1111/j.14401746.2011.06850.x, 2012.

[60] D. Norimura, H. Isomoto, N. Yamaguchi, Y. Akazawa, K. Ohnita, S. Shikuwa, F. Takeshima and K. Nakao, "Analysis of the tumor color patterns of early esophageal cancer using an autofluorescence imaging video endoscopy.," Surg Laparosc Endosc Percutan Tech., p. 10.1097/SLE.0b013e31822a327e., 2011.

[61] C. D’Orsi, E. Sickles, E. Mendelson, E. Morris and e. a. , ACR BI-RADS ${ }^{\circledR}$ Atlas, Breast 
Imaging Reporting and Data System, VA: Reston, American College of Radiology, 2013.

[62] P. Crystal, M. Koretz, S. Shcharynsky and e. a. , "Accuracy of sonographically guided 14-gauge core-needle biopsy: Results of 715 consecutive breast biopsies with at least two-year follow-up of benign lesions," pp. 33:47-52, 2005.

[63] R. Alfano, "In Advances in optical biopsy and optical mammography," New York Academy of Sciences, 1998.

[64] L. Hoagland and R. Hitt, Techniques for ultrasound-guided, percutaneous coreneedle breast biopsy, Boston, MA: J of practical med imaging and management, 2013.

[65] A. Kalitzeos, A. Zam, F. Stelzle, E. Hahn, M. Raithel and A. Douplik, "Enhancement of Cancerous/Normal Tissue Contrast viaCombined White Light and Fluorescence Image Processing initial," Biomedical Optics, p. 10.1117/12.831771, 2009.

[66] T.871: Information technology, "Digital compression and coding of continuous-tone still images," 2012.

[67] Breastcancer.org, Your Guide to the Breast Cancer Pathology Report, Genetech, 2016.

[68] B. Brancato, E. Crocetti, S. Bianchi, S. Catarzi, G. Risso, P. Bulgaresi, F. Piscioli, M. Scialpi, S. Ciatto and N. Houssami, "Accuracy of needle biopsy of breast lesions visible on ultrasound: audit of fine needle versus core needle biopsy in 3233 consecutive samplings with ascertained outcomes," Breast, pp. 21(4):449-54., August 2012.

[69] Z. Movasaghi, S. Rehman and I. U. Rehman, "Raman Spectroscopy of Biological Tissues," Appl. Spectrosc. Rev, pp. 42, 493-541, 2007. 